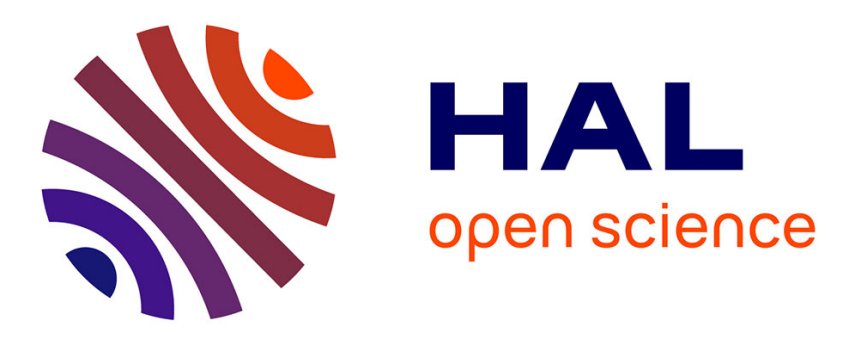

\title{
Progress in developing cationic vectors for non-viral systemic gene therapy against cancer.
}

Marie Morille, Catherine Passirani, Arnaud Vonarbourg, Anne Clavreul, Jean-Pierre Benoit

\section{- To cite this version:}

Marie Morille, Catherine Passirani, Arnaud Vonarbourg, Anne Clavreul, Jean-Pierre Benoit. Progress in developing cationic vectors for non-viral systemic gene therapy against cancer.. Biomaterials, 2008, 29 (24-25), pp.3477-96. 10.1016/j.biomaterials.2008.04.036 . inserm-00353462

\section{HAL Id: inserm-00353462 https://www.hal.inserm.fr/inserm-00353462}

Submitted on 15 Jan 2009

HAL is a multi-disciplinary open access archive for the deposit and dissemination of scientific research documents, whether they are published or not. The documents may come from teaching and research institutions in France or abroad, or from public or private research centers.
L'archive ouverte pluridisciplinaire HAL, est destinée au dépôt et à la diffusion de documents scientifiques de niveau recherche, publiés ou non, émanant des établissements d'enseignement et de recherche français ou étrangers, des laboratoires publics ou privés. 
Progress in developing cationic systems for non-viral vector systemic gene therapy against cancer

Morille M., Passirani C., Vonarbourg A., Clavreul A., and Benoit J.P.*

Inserm U646, Ingénierie de la Vectorisation Particulaire, Université d'Angers, 10, rue André Boquel, 49100 - Angers, France.

*Corresponding author. Tel. : 33 (0)2 417358 58. Fax : 33 (0)2 41735853

E-mail address: jean-pierre.benoit@univ-angers.fr 


\section{Abstract:}

Initially, gene therapy was viewed as an approach for treating hereditary diseases, but its potential role in the treatment of acquired diseases such as cancer is now widely recognized. The understanding of the molecular mechanisms involved in cancer and the development of nucleic acid delivery systems are two concepts that have led to this development. Systemic gene delivery systems are needed for therapeutic application to cells inaccessible by percutaneous injection and for multi-located tumor sites, i.e. metastases. Non-viral vectors based on the use of cationic lipids or polymers appear to have promising potential, given the problems of safety encountered with viral vectors. Using these non-viral vectors, the current challenge is to obtain a similarly effective transfection to viral ones. Based on the advantages and disadvantages of existing vectors and on the hurdles encountered with these carriers, the aim of this review is to describe the "perfect vector" for systemic gene therapy against cancer. 


\section{Summary}

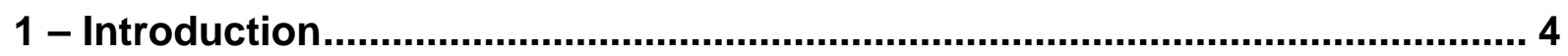

2 - Non viral vectors: current cationic systems ……....................................... 6

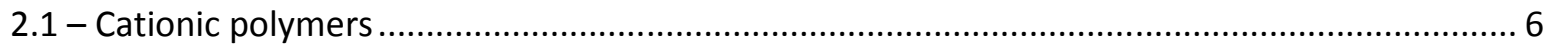

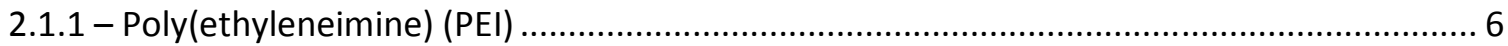

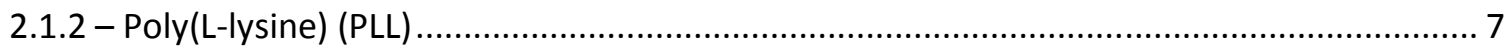

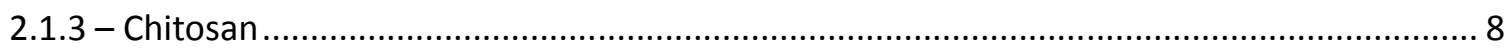

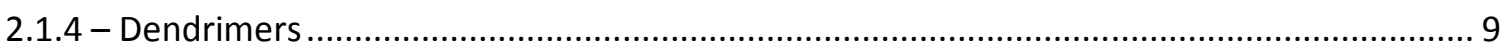

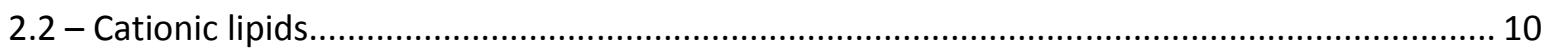

3 - Principal hurdles for cationic systems .................................................... 13

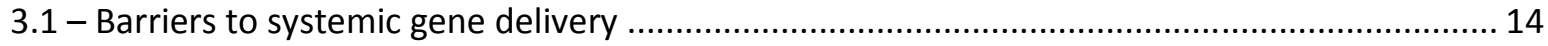

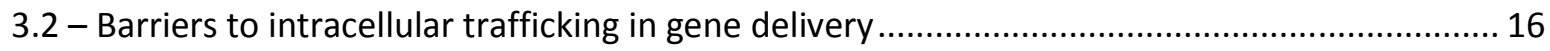

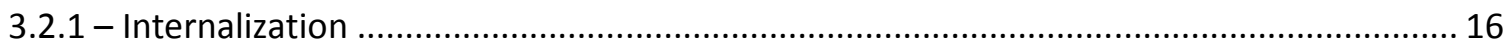

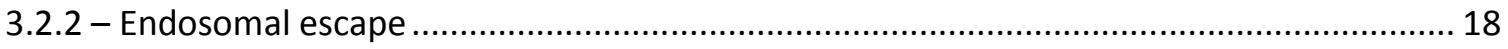

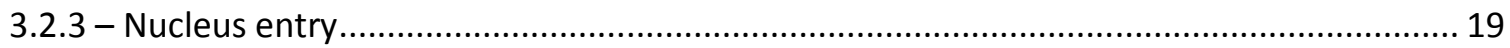

4 - Strategies to improve systemic delivery and intracellular trafficking of cationic

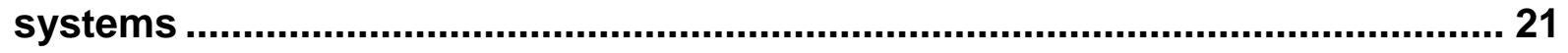

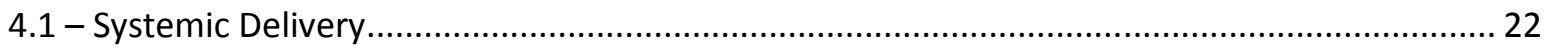

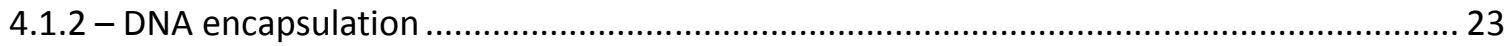

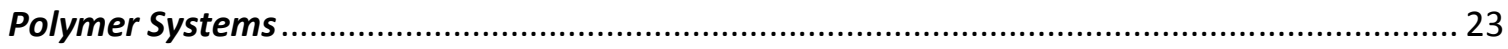

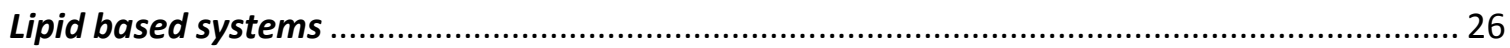

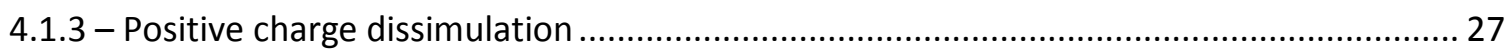

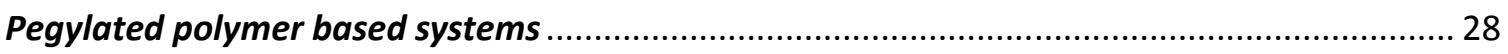

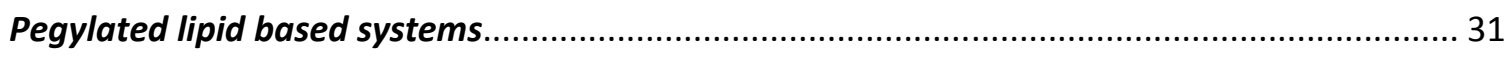

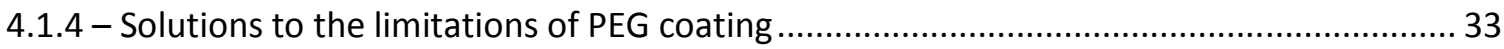

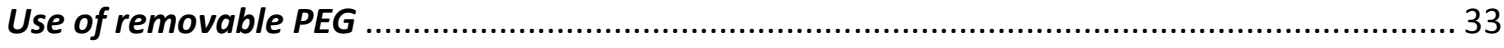

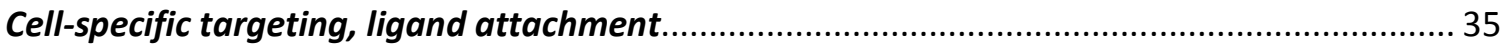

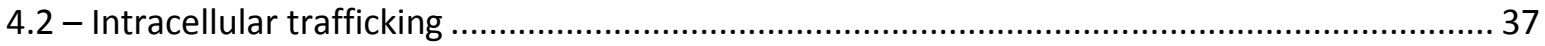

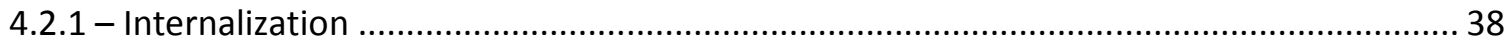

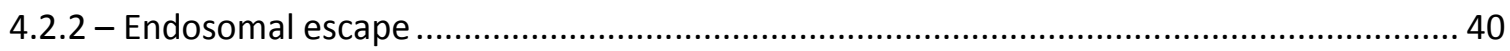

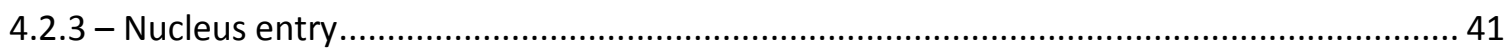




\section{1 - Introduction}

Cancer has become the first killer in developing country and is on the verge of becoming the first cause of death in industrialized countries. Due to its invasive, aggressive growth profile as well as the complex mechanisms involved in cancer development and propagation, classical treatments such as surgery, chemotherapy and radiotherapy are still insufficiently effective in many cases, and are often up against resistant and infiltrating tumors. New anticancer strategies are thus urgently required.

A better understanding of the genes involved in the development and growth of cancer is leading to new approaches to treat this disease. Oncogenes and tumor suppressor genes, disturbed in most cancers, play a crucial role in the beginning and growth of the cancerous processes [1]. The approach of gene therapy provides a promising tool to eradicate this disease by treating it at its source. It can be particularly advantageous in that a relatively short expression of therapeutically active proteins may be sufficient to eradicate tumors, unlike genetic diseases such as cystic fibrosis in which there is need for long-term expression [2]. Interestingly, between 1989 and 2004, cancer was the first candidate for gene therapy clinical trials (66\% of all gene therapy trials) [3] (http://www.wiley.co.uk/genmed/clinical/). In the field of cancer gene therapy, four major targets have to be reached by vectors: turning off oncogene expression, enhancing tumor suppressor expression to induce apoptosis of cancer cells, inhibiting neoangiogenesis, and stimulating immune system against tumors cells.

Historically, there have been three different applied approaches to gene delivery. The first approach consists of the use of naked DNA. Direct injection of free DNA to the tumor site has been shown to produce high levels of gene expression and the simplicity of this approach led to its use in a number of experimental protocols $[4,5]$. This strategy appears to be limited to tissues that are easily accessible by direct injection such as the skin and 
muscles [6] and is unsuitable for systemic delivery due to the presence of serum nuclease. The second approach involves using genetically-altered viruses. Viral vectors are biological systems derived from naturally evolved viruses capable of transferring their genetic materials into the host cells. Many viruses including the retrovirus, adenovirus, herpes simplex virus (HSV) and adeno-associated virus (AAV) have been modified to eliminate their toxicity and maintain their high capacity for gene transfer [7] hence presenting various advantages [8-10]. Viral vectors are very effective in achieving high efficiency for both gene delivery and expression. However, the limitations associated with viral vectors, in terms of safety, immunogenicity, low transgene size and high cost, has encouraged researchers to focus on alternative systems. The third approach for delivery systems concerns non-viral vectors, which are mainly of a cationic nature: cationic polymers and cationic lipids. They interact with negatively charged DNA through electrostatic interactions leading to polyplexes and lipoplexes, respectively. The advantages associated with these kind of vectors include their large scale manufacture, their low immunogenic response, the possibility of selected modifications and the capacity to carry large inserts (52 kb) (Table 1) [11, 12]. While the transfection efficiency of non-viral vectors is still lower than for their viral counterparts, a number of adjustments (e.g. ligand attachment) could improve this category of carriers which are, thus far, believed to be the most promising of gene delivery systems. Nonetheless, this class of vectors has to be modified to make systemic delivery possible. To date, systemic administration has resulted in a toxic response (linked to their positive charge), incompatible with clinical applications.

Currently, the main objective in gene therapy via a systemic pathway is now the development of a stable and non-toxic gene vector that can encapsulate and deliver foreign genetic materials into specific cell types such as cancerous cells with the transfection efficiency of viral vectors. 
In parallel to existing review in non viral gene delivery against cancer ([13-16]), the aim of this work is to provide a non-exhaustive list of the cationic vectors currently developed for systemic delivery (for other administration pathways see reviews [17-19]). The obstacles to their systemic injection and cell trafficking will be described. The possible strategies to overcome these problems will be argued thereafter.

\section{2 - Non viral vectors: current cationic systems}

2.1 - Cationic polymers

\subsection{1 - Poly(ethyleneimine) (PEI)}

PEI can be synthesized in different lengths, be branched or linear (Fig.1), and undergo functionalized group substitution or addition. It is a versatile polymer which has a privileged place in the components of non-viral gene delivery, due to its superior transfection efficiency in a broad range of cell types compared to other systems described later. PEl polymers are able to successfully complex DNA molecules, leading to homogeneous spherical particles (Table 1) [20]. Studies showed that linear PEI with low molecular weight were the most efficient in transfection and the least cytotoxic [21]. Non-protonated amines with different pKa values gave the $\mathrm{PEI}$ a buffering effect in a wide range of $\mathrm{pH}$ levels. This buffering property enabled the PEI to escape from the endosome due to the mechanism known as the 'protosponge' effect (enlightened later in this review §3.2.2) [22]. However, the high amount of 
positive charges and their non biodegradability resulted in fairly high toxicity of PEI polymers in vivo $[23,24]$.

\subsection{2 - Poly(L-lysine) (PLL)}

Because of its peptide structure, PLL has a biodegradable nature, which is an advantage for in vivo use (Fig.1). Until 2000, PLL was one of the most used cationic polymers for DNA delivery (table1) $[25,26]$. Having a low molecular weight (less than $3 \mathrm{kDa}$ ) PLL cannot form stable complexes [27]. Furthermore, it would appear that high molecular weight PLL is more suitable for gene delivery via systemic injection, with PLL 211kDa/DNA complexes displaying levels in the blood up to 20 -fold higher after 30 minutes compared to PLL 20kDa/DNA complexes. Indeed, the complexes formed with low molecular weight PLL, are fixed by the complement system in vitro, are less soluble in vivo (aggregation), and are thus rapidly removed by the Kupffer cells of the liver. Destabilization of this constructs in the blood is described as a possible mechanism for their removal of the blood circulation.[28].

The PLL/DNA polyplexes are internalized in a way comparable to PEI/DNA complexes, but their transfection efficiency is weak (due to a lack of amino groups allowing endosomolysis [15], cf $\S 3.2 .2)$.

A degradable PLL analogue, poly(a-[4-amino-butyl]-L-glycolic acid) (PAGA) showed significantly higher transfection efficiency than PLL, while no measurable cytotoxicity was detected [29]. For example, PAGA was used to deliver plasmid DNA (pDNA) encoding murine interleukin 10 (IL-10), via systemic injection in NOD (Non Obese Diabetic) mice: the peak level of IL-10 expression was achieved at Day 5 after injection and gene expression 
lasted for more than 9 weeks [30]. The combined systemic administration of plasmid encoding IL-4 and IL-10 using PAGA to NOD mice also showed good expression levels [31].

\subsection{3 - $\underline{\text { Chitosan }}$}

Chitosan is a biodegradable and biocompatible linear aminopolysaccharide composed of 1-4 linked N-acetyl-D-glucosamine and D-glucosamine subunits (Fig. 1), obtained by deacetylation of chitin (a polysaccharide found in the exoskeleton of crustaceans and insects [32] [33]). It can complex pDNA and is capable of forming stable, small (20-500 nm) particles depending on the molecular weight and the degree of deacetylation (Table 1) [34]. Its cationic polyelectrolyte nature provides strong electrostatic interaction with mucus, negatively charged mucosal surfaces and other macromolecules such as DNA [33, 35]. This cationic polymer provides protection against DNase degradation that is comparable to PEl's one, and displays a significantly better biocompatibility [36]. Consequently, several groups have conducted studies using chitosan/DNA nanoparticles, including use of galactosylated chitosan [37], galactosylated chitosan-graft-poly(vinylpyrrolidone) (PVP) [38], trimethylated chitosan oligomers [39], N-dodecylated chitosan [40], deoxycholic acid modified chitosan [41] or ligand attached chitosans for targeting cell membrane receptors [42].

Cellular RNA interference machinery is now used in cancer gene therapy, to turn off oncogene expression, for instance. This strategy is promising but there is a strong need to find an efficient vector enhancing the bioavailability of these small interference RNA molecules (siRNA). Chitosan nanoparticles have therefore been used to transport siRNA. Katas et al. [43] first studied the behavior of interaction between siRNA and chitosans given that the structure and size of siRNA is quite different to that of pDNA. The ability of these nanoparticles to mediate gene silencing was assessed in vitro on CHO K1 and HEK 293 
cells. Furthermore, an in vitro study revealed that the transfection efficiency of siRNA depended on its association with chitosan. Indeed, entrapping siRNA using ionic gelation showed a better biological effect than simple complexation or siRNA adsorption onto the chitosan nanoparticles. This finding might be attributed to a strong binding between the chitosan and siRNA (determined by gel retardation assay) and a better loading efficiency when using ionic gelation [43].

\subsection{4 - Dendrimers}

Dendrimers are spherical, highly branched polymers. Dendrimers (from the greek dendron: 'tree' and meros: 'part') [44] are specific in that they have a hierarchical, tridimensional structure. The heart of the molecule provides a central point from which monomers will ramify in a well-ordered and symmetrical manner. The tree-like construction is made by the repetition of the same sequence of reactions until the formation, at the end of every reaction cycle, of a new generation with an increased number of identical branches. The most currently used dendrimers are polyamines, polyamides or polyesters, but the most commonly encountered is polyamidoamine (PAMAM) because of its high transfection efficiency (Fig.1) (Table 1) [45-47]. Dendrimers bear primary amine groups on their surface and tertiary amine groups inside. The primary amine groups participate in DNA binding, compact it into nanoscale particles and promote its cellular uptake, while the buried tertiary amino groups act as a proto-sponge in endosomes and enhance the release of DNA into the cytoplasm. The size and diameter of dendrimers have an influence on their transfection efficiency. Thus, the transfection efficiency obtained with high generation dendrimers (10) is clearly superior to low generation dendrimers (5) [48]. Partially degraded PAMAM dendrimers are reported to have more flexible structures than intact dendrimers and therefore to interact 
more efficiently with DNA [49]. A fragmentation step consisting of hydrolytic cleavage of the amine bonds is needed to enhance the transfection efficiency [46, 49-51].

\section{2 - Cationic lipids}

Cationic lipids represent the second group of synthetic vectors commonly used in gene delivery. Since first being use for gene therapy in 1987 by Felgner et al. [52], numerous cationic lipids (also called cytofectins or lipofection reagents) have been synthesized and used for delivery in cell culture, animals, and patients enrolled in phase I and II of clinical trials (http://www.wiley.co.uk/genmed/clinical/). Cationic lipids are technically simple and quick to formulate, readily available commercially, and may be tailored for specific applications.

Cationic lipids are made up of a cationic head group attached by a linker to a lipid hydrophobic moiety. The positively charged head group is necessary for the binding of nucleic acid phosphate groups. All cationic lipids are therefore positively charged amphiphile systems. They can be classified into various subgroups according to their basic structural characteristics (Fig.2):

(1) monovalent aliphatic lipids characterized by a single amine function in their headgroup e.g. $\mathrm{N}$ [1-(2,3-dioleyloxy) propyl]-N,N,N-trimethylammonium chloride (DOTMA), 1,2-dioleyl-3-trimethylammonium-propane (DOTAP), N-(2-hydroxyethyl)-N,N-dimethyl-2,3bis(tetradecyloxy-1-propanaminium bromide) (DMRIE),

(2) multivalent aliphatic lipids whose polar headgroups contain several amine functions such as the spermine group e.g. dioctadecylamidoglycylspermine (DOGS), 
(3) cationic cholesterol derivatives e.g. $3 \beta-\left[N-\left(N^{\prime}, N^{\prime}-\right.\right.$ dimethylaminoethane)carbamoyl]cholesterol (DC-Chol), bis-guanidium-tren-cholesterol (BGTC).

In general, reports indicate that the myristoyl (C14) chain is optimal for transfection (compared to C16 or C18 compounds), followed by the oleoyl chain $(\mathrm{C} 18: 1)[53,54]$. Increasing the aliphatic chain length for amphipathic compounds of this type is known to increase both the phase transition temperature and the bilayer stiffness of the resulting vesicles, and having a stiff bilayer is unsuitable for membrane fusion (which is an important step in DNA delivery mechanism) [53].

Adding DNA to cationic liposomes results in either lamellar or inverted hexagonal phase structure (Fig.3). The lamellar form is a condensed and globular structure, consisting of DNA monolayers, characterized by uniform inter-helical spacing, sandwiched between cationic lipid bilayers [55], while the inverted hexagonal phase structure consists of DNA coated with cationic lipid monolayers arranged on a two-dimensional hexagonal lattice [56, 57]. For transfection application, cationic lipids are often mixed with so-called helper lipids, like DOPE (1,2-dioleyl-sn-glycerol-3-phosphoethanolamine) (Fig.2) or cholesterol, both potentially promoting conversion of the lamellar lipoplexe phase into a hexagonal structure, which is known to improve transfection efficiency $[58,59]$. It is worth noting that the ratio and combination of cationic/helper lipids are important factors for transfection efficiency and toxicity $[60,61]$. In 1993, Zhu et al. [62] reported a relatively low in vivo gene expression by intravenous (i.v) injection of pDNA complexed with DOTMA/DOPE liposomes. Additionally, DMRIE and DC-Chol were tested in clinical trials but the resulting therapeutic effects were disappointing and the formulation was hampered by toxicity [63]. After these first in vivo studies, several studies showed that various factors could enhance gene expression in vivo: a high cationic charge [64-67], cholesterol-containing liposomes [66, 68-70], a high dose of pDNA $[64,65,69,71-73]$, the absence of non methylated CpG (dinucleotides composed of a 
cytosine followed by a guanine) otherwise recognized by the human immune system and that induces an inflammatory cytokine expression [74, 75].

Systemic application of an improved extruded DOTAP/cholesterol cationic liposome formulation loaded with therapeutic tumor suppressor p53 pDNA resulted in successful treatment of primary and disseminated lung cancers in a mouse model [76]. More recently, a polycationic sphingolipid analogue was synthesized, containing a spermine headgroup. Interestingly, it was shown that the capacity of this compound to deliver antisense oligodeoxynucleotides (AS-ODN) into cells, as reflected by an efficient anti-bcl-2 (apoptosis inhibitor) effect, was superior to that of vectors prepared from DOTAP or DC-Chol [77].

Lipoplexes have recently been applied for siRNA delivery in vivo [78-80]. One example is the silencing of the hdm2 oncogene in p53 dependent human breast cancer [81]. Cationic lipoplexes (prepared with cationic liposome containing 2-O-(2- diethylaminoethyl)-carbamoyl1,3-O-dioleoylglycerol and egg phosphatidylcholine) formulations of siRNA targeting bcl-2 showed antitumor activity in two different tumor mouse models [82]. Peritumoral administration of these lipoplexes inhibited tumor cell growth of subcutaneously established prostate cancer, whereas an i.v injection had strong antitumor activity in a mouse model of liver metastasis or lung carcinoma.

Landen et al. [83] encapsulated siRNA into neutral dioleoylphosphatidylcholine (DOPC) liposomes, which delivered siRNA efficiently into the tumor but also into other major organs, after i.v infusion. In nude mice bearing intraperitoneal ovarian tumors, DOPCencapsulated siRNA targeting the oncogene EphA2 was highly effective in reducing in vivo EphA2 expression 2 days after a single injection. Four weeks of treatment with EphA2 siRNA liposomes reduced tumor growth by about $50 \%$. These results were reduced again by $15 \%$ when administered in association with the anti-cancer drug paclitaxel. 
Another new approach used in clinical trials for cancer gene therapy is to employ immunological strategies, using the transfer of gene for cytokines. This concept was further developed toward the systemic application of lipoplexes. Dow et al. [84] evaluated repeated i.v delivery of IL-2 pDNA lipoplexes [cholesterol / DOTIM (1-[2-(oleoyloxy)ethyl]-2-oleyl-3-(2hydroxyethyl)imidazolinium chloride)] (Fig.2) in a pre-clinical phase I study in 20 dogs with chemotherapy-resistant osteosarcoma metastases. These injections were well tolerated, resulting in detectable IL-2 transgene expression in lung tissues and significantly increased overall survival times in treated dogs compared with historical controls at the same stage of disease. Three of the 20 dogs experienced partial or complete regression of lung metastases.

\section{3 - Principal hurdles for cationic systems}

For disseminated cancer diseases, such as many cancer forms, treatment needs to be administered systemically and therefore must be targeted to the cancer cells. Systemic targeting of tumors via the bloodstream is a real challenge: the cationic systems have to survive in the bloodstream without being degraded or captured by cellular defense mechanisms [85-90]. Once at the tumor site, they have to extravasate into the tissue and bind specifically to the target cells. After their cellular internalization, intracellular barriers (endosomal escape, cytoplasm trafficking, nucleus entry) are additional hurdles, in which each of the listed steps can be a major bottleneck for the efficiency of such a gene delivery system (Fig.5) [91, 92]. 
3.1 - Barriers to systemic gene delivery for cationic systems

The main obstacles to the use of polyplexes or lipoplexes via systemic delivery are their aggregation, instability, toxicity and their propensity to be captured by the mononuclear phagocyte system (MPS).

Numerous biodistribution studies show an accumulation of cationic systems in the lungs, liver and spleen. This accumulation can be explained by the fact that these non-viral gene delivery systems at therapeutic doses require high concentrations of lipoplexes or polyplexes and these positively charged particles readily aggregate as their concentration increases. These aggregates which are generally ineffective gene delivery agents can be toxic due to embolization of the particulates in the lung. Classical studies of colloid behaviour assume that particles of similar charge, possessing an electrostatic repulsion that is greater than the Van der Waals forces, are stable against aggregation. But in the case of DNAcationic (lipid or polymer) complexes, this classical electrostatic stabilization model seems to be inadequate. A more complex model such as that of bridging gap between particles by extent polymer loops or by collision between electrostatic surfaces of opposite charges on the particles, may be required [50]. The surface charge of the complex depends on the nature of the condensing agent and also on the ratio of the condensing agent to DNA. Numerous studies expose that the stability of DNA-complexes under physiological buffer conditions is an important consideration in the design of particles, with particles presenting no aggregate showing the best transfection efficiency [50].

Furthermore, an accumulation of gene delivery vectors in the lungs could be explained by the electrostatic interaction of cationic systems with negatively charged erythrocyte membranes [93]. In the same way, positively charged particles can be opsonized with plasma proteins such as immunoglobulin M, complement $\mathrm{C} 3$ and proteins of the coagulation cascade 
[20] leading to their rapid clearance by phagocytic cells of MPS in the liver, spleen, lungs, and bone marrow [94]. This opsonization can also activate the complement system, one of the innate immune mechanisms against 'foreign' particles within the bloodstream [95], which in turn activates the phagocytosis and initiates an inflammatory response against positively charged particles [96]. The clearance rate of these vectors from the circulatory system in fact depends on their physicochemical surface characteristics. To be 'stealthy' (undetectable by macrophages) [97], vectors have to be as small and neutral as possible [98].

At the same time, other serum proteins, in particular albumin, lipoproteins (HDL and LDL) and macroglobulin, interact with cationic lipid/nucleic acid complexes, and can alter the complex diameter and zeta potential (from positive to negative values) [99]. These interactions can provide destabilization of the system and nucleic acids release which could be recognized by Toll like receptor 3 (TLR3) (which recognize double stranded RNA [100]), TLR7 and TLR8 (single stranded RNA[101]) or TLR9 (bacterial DNA[102]) expressed in B cells and plasmacytoid dendritic cells, inducing cytokine production at the origin of their toxicity [103]. A dose-dependent toxicity of different kinds of lipoplexes (GL-67 (N4-spermine cholesterylcarbamate) and GL-62 (N1-spermine cholesterylcarbamate), DMRIE (1,2dimyristoyloxypropyl-3-dimethyl-hydroxyethylammonium bromide), DOTMA:DOPE) was observed, for instance, after injection in a mouse presenting hair erection and lethargy. Clinical studies have shown dose-dependent haematological and serological changes typified by profound leukopenia, thrombocytopenia, and elevated levels of serum transaminases indicative of hepatocellular necrosis [103].

However, this strong immune response could be used to develop plasmid DNA vaccines for cancer [104]. As an example, systemic administration of lipoplexes [DOTIM (1[2-(oleoyloxy)ethyl]-2-oleyl-3-(2-hydroxyethyl)imidazolium chloride) and cholesterol associated to DNA] can trigger strong activation of the innate immune response and release 
of high concentrations of Thelper type 1-cytokines like IL-12 and interferon (IFN- $\mathrm{\gamma}$ ) providing an enhancement of the immune reaction against tumor cells [104]. Additionally, systemic administration of cationic lipid-DNA complexes can display potent antitumor activity depending on both NK cells and IFN-y. [105, 106]. Moreover, lipoplexe vaccination can elicit large numbers of functionally active and tumor specific infiltrating CD8+ T cells. Such an advance could be considered for patients whose tumors express known tumor antigens including melanoma, renal cell carcinoma, prostate cancer and colon cancer. This approach needs to be applied clinically and an optimization of the balance between induction of $\mathrm{T}$ cellmediated antitumor immunity and induction of toxicity by over-stimulation of innate immune responses needs to be achieved $[105,106]$.

3.2 - Barriers to intracellular trafficking in gene delivery

Once the vector is at the tumor site, the tumor matrix, consisting of collagen and other proteins, is a further barrier to gene delivery, as the target cannot easily be reached because of limited diffusion of the vector within the tissue [107]. Depending on the type of tumor, the influence of the extracellular matrix composition and structure on macromolecule mobility can vary significantly. For exemple, a 5- to 10-fold faster diffusion of large molecules (such as non-specific IgM, FITC-dextran 2,000,000 MW or liposomes) was evidenced in cranial window tumor models compared to dorsal chamber tumor models [107]. Moreover, following this extracellular barrier, there are several intracellular hurdles (Fig.4) as described below.

3.2.1 - Internalization 
The pathway followed by the cationic carriers, from the exterior of the cell to the nucleus, is not yet fully understood, but the fusion of vesicles with the plasma membrane is perceived as a better route, since it avoids the endolysosomal compartment (with its acidic environment resulting in DNA/RNA degradation). However, studies of electron and fluorescence microscopy have shown that lipoplexes and polyplexes can be detected in intracellular vesicles beneath the cell membrane, suggesting that they enter cells by endocytosis and will thus be directed towards the endolysosomal compartment [108-110].

There are a multitude of endocytic pathways that can be processed by the carrier systems: clathrin-mediated endocytosis via coated pits (adsorptive or receptor mediated), lipid raft mediated endocytosis ( caveolae-mediated or not), phagocytosis, macropinocytosis (Fig.4) [111, 112].

The predominant way of entry of cationic gene delivery systems seems to be by nonspecific adsorptive endocytosis followed by the clathrin-coated pit mechanism, because negatively charged glycoproteins, proteoglycans and glycerophosphates, present on the cell membrane, are able to interact with the positively charged systems $[113,114]$. Using specific inhibitors of different endocytosis pathways, Rejman et al. [115] conclude that lipoplexe (DOTAP/DNA) uptake can be proceeded only by clathrin-mediated endocytosis, while polyplexes (PEI/DNA) can be taken up by two mechanisms, one involving caveolae and the other clathrin-coated pits. However, the internalization pathway seems to be dependent on the system used and the cells to transfect $[116,117]$. Carrier systems containing a specific targeting moiety, which are specifically recognized by a cell surface receptor could enter cells via both adsorptive endocytosis and receptor-mediated endocytosis [118].

Macropinocytosis can also mediate the uptake of cationic carriers because of its ability to internalize large structures such as bacteria [119]. Phagocytosis of lipoplexes and 
polyplexes, even in cell lines that are not professional phagocytes, has also been evidenced $[120,121]$.

The relative contribution of each pathway in the internalization of synthetic vectors is poorly defined, given the large variety of carriers [122]. Therefore, factors such as cell membrane composition or surface charge and the size [123] of complexes may influence the balance in favour of either one or the other pathway.

\subsection{2 - Endosomal escape}

If the gene delivery system enters the cells via an endocytosis pathway, endosome capture will represent a major barrier to efficient transfection. In order to be effective, the vector, or at least its content, has to be released from this compartment, preferably at an early stage.

The mechanisms involved in endosomal release of DNA by cationic polymer-based vectors are unsure. Two hypotheses have been suggested to explain this escape. The first one is based on the idea that a physical disruption of the negatively charged endosomal membrane occurs on direct interaction with the cationic polymer. Such a mechanism has been suggested for both PAMAM dendrimers and PLL [124]. Interestingly, this mechanism seems to depend on the target membrane composition (cell type). The second hypothesis used to explain endosomal disruption by cationic polymers with ionizable amine groups has been termed the "proton-sponge" hypothesis (Fig.5) [22]. Endosomal membranes possess an ATPase enzyme that actively transports protons from the cytosol into the vesicle resulting in acidification of the compartment [125]. The proton-sponge hypothesis assumes that polymers such as PEI and PAMAM, containing a large number of secondary and tertiary amines, can buffer the $\mathrm{pH}$, causing the ATPase to transport more protons to reach the 
desired $\mathrm{pH}$. The accumulation of protons in the vesicle results in an influx of counter ions which causes osmotic swelling and rupture of the endosome membrane, in turn releasing the polyplexes into the cytoplasm $[22,126,127]$.

In the case of cationic lipid-based vectors, another model has been proposed for local endosomal membrane destabilization, in which electrostatic interactions between the cationic lipids and the endosome membrane induce the displacement of anionic lipids from the cytoplasm-facing monolayer of the endosome membrane, by way of the so called flip-flop mechanism (Fig.5). The formation of a neutral ion pair between anionic lipids present in the endosomal membrane and the cationic lipids of the vector, will then cause subsequent decomplexation of the DNA and finally its release into the cytoplasm [128, 129]. Additionally, non-cationic helper lipids such as neutral DOPE facilitate membrane fusion and help destabilize the endosomal membrane $[60,130,131]$. It is indeed known that DOPE has a tendency to form an inverted hexagonal phase, often observed when membranes are fusioned.

Interestingly, it has been suggested that transfection with the multivalent lipopolyamine DOGS $[22,132]$ or with BGTC containing a tertiary amine with a low pK [133] involves escape from the endosome to a similar process to that proposed earlier for cationic polymers PEI: the "proton-sponge" mechanism.

Despite these escape hypotheses, the majority of gene delivery systems seems to be stopped at this stage.

3.2.3 - Nucleus entry 
After endosomal escape, the nucleic acid must traffic through the cytoplasm and enter the nucleus. We could hypothesize that in case of the use of siRNA, this hurdle can be overcome since the interference mechanism arises in the cytoplasm. The mobility of large molecules, such as pDNA, is extremely low in the cytoplasm, making them an easy target for cytoplasmic nucleases [134]. Lechardeur et al. [135] reported that the half-life of a naked pDNA in the cytoplasm of Cos7 and HeLa cells is $50-90$ minutes. Thus pDNA has to be both protected and also available to enter the nucleus.

An important factor in nucleic acid transport through the cytoplasm is the rate of mobility which depends on the size and spherical structure of the molecule (circular plasmid DNA > linear DNA) [28]. But in the case of DNA complexed with gene delivery systems, the state of DNA when present in the cytoplasm is poorly documented and almost unknown. Indeed, the DNA molecules could be free or still associated to their carrier, and in a compacted state. This compaction could lead to increased cytosolic mobility or could offer increased stability against cytoplasmic nucleases. In the case of polyplexes, the microinjection of PEl/pDNA complexes resulted in a 10-fold higher levels of transgene expression compared to naked DNA, and showed that the enhanced expression may be the consequence of increased cytoplasmic mobility, due to the smaller size of the compacted DNA [136].

It is known that plasmids that are microinjected in the cytoplasm of cultured cells are poorly expressed whereas those that are microinjected directly in the nucleus are highly expressed [137]. These results indicate that nucleus entry is another important barrier (the final one) for efficient transfection. Indeed, DNA needs to access transcription machinery which is present inside the nucleus.

The nuclear envelope, a double membrane, is interrupted by large protein structures called nuclear pore complexes (NPC). These proteins allow the passage of molecules up to 
$9 \mathrm{~nm}$ in size (40 to $60 \mathrm{kDa})$, but in the case of larger macromolecules, the transfer needs shuttle molecules and is energy-dependent [138]. The NPC is able to mediate the transport of ions, small molecules, proteins, RNA, and ribonucleoproteins in and out of the nucleus. Specific sequences on proteins expected to enter the nucleus, named Nuclear Localisation Sequence (NLS) allow intracellular protein trafficking toward the nuclear pores [139]. The first NLS described was the derived sequence of the simian cancer virus large T antigen [140]. These NLS are recognized in the cytoplasm by a soluble protein, importin- $\alpha$ [139]. The complex of NLS/importin- $\alpha$ connects to another protein, importin $\beta$, and this trimeric complex then docks at the NPC and can enter the nucleus (Fig. 6) [141].

Cytoplasm mixing and the loss of nuclear membrane during mitosis could be a way to overcome this problem. Consistent with this hypothesis, gene transfer in cultured cells has been shown to be greatly enhanced by mitotic activity for both lipoplexes [142, 143] and polyplexes [143]. This would mean that non dividing cells are rarely transfected, and this could be a positive point for targeting tumoral cells, especially in the brain where healthy cells have no or low dividing activity.

\section{4 - Strategies to improve systemic delivery and intracellular trafficking of cationic systems}

As seen previously (§ 3.1), systemic delivery represents a daunting hurdle in gene therapy and nowadays there is no general approach to systemic gene delivery. However, numerous efforts have been made to obtain effective and stable gene delivery systems and interesting and promising solutions have been found, as described below (Table 2). 


\section{1 - Systemic Delivery}

\subsection{1 - DNA condensation in ternary system}

These systems consisting of hybrid constructs with different polymers or between polymers and lipids have been synthesized by the precondensation of nucleic acids with a number of different possible polycationic agents. They have been developed to allow a smaller size and a better stability of the colloids during their stay in the blood.

An approach to ameliorate the complexation of nucleic acids developed by Hwang et al. [144] consists in associating cationic polymers including cyclodextrin rings within them. The cationic nature enables DNA complexation whereas cyclodextrin rings allow PEG binding.

In the same way, the interaction of pDNA with protamine sulfate, followed by the addition of DOTAP cationic liposomes, allow the creation of LPD (liposome/protamine/DNA) to take place. Protamine is a naturally occurring polycation which condenses DNA in the head of spermatozoa. The nuclear localizing property of protamine makes it particularly attractive for transfection applications. Also, protamine sulfate is a small defined peptide system (4-4.25 kDa), that possesses a high affinity for DNA structure [145] and contains a short runs of basic amino acids known to act as NLS [89]. This small polycationic agent would be expected to show low probability of immunogenic responses in the target tissue due to the absence of aromatic amino acids and the lack of a rigid structure [146]. Compared to classic DOTAP/DNA lipoplexes, LPD offers better nuclease protection and gives consistently higher gene expression in mice via tail vein injection [147]. The E1A protein has been demonstrated to elicit antitumor effects through various mechanisms, including the induction of apoptosis and also sensitization to chemotherapeutic agents and radiation. The systemic 
delivery of LPD lipopolyplexes, encoding the E1A protein, to human xenograft tumor models for breast, head and neck cancer, resulted in reduced tumor growth in both models [148]. The combination of systemic E1A pDNA therapy and paclitaxel chemotherapy strongly enhanced therapeutic effects and dramatically repressed tumor growth, in the case of a breast cancer model [149].

Several ternary systems, using other cationic condensing molecules, have been described in the past few years including systems based around mu peptide [150, 151], PEI [152].[153], PLL [154, 155], spermidine [156], lipopolylysine [110], histone proteins [157, 158], chromatin proteins [159], human histone derived peptides [90], oligo-L-lysine [160, 161], L-lysine containing synthetic peptides [162] and a histidine/lysine (H-K) copolymer [163]. For example, a significant decrease in p53 protein biosynthesis in HepG2 and hepatoma 2.2.15 cells was only seen with PEI/DOTAP-Chol formulation but not with cationic liposomes or PEI alone [153]. However, these systems are, like lipoplexes, positively charged and as seen previously (§3.1), when injected systemically, they risk to be sensitive to plasmatic proteins that lead to their destabilization and consequently the confrontation of nucleic acids to nucleases and/or their clearance by the MPS. The first in vivo results concerning LPD confirmed this hypothesis [64, 164].

\subsection{2 - DNA encapsulation}

\section{Polymer Systems}

In parallel to the studies based on polyplexes, nanoparticle-based systems were developed. Nanoparticles are colloidal drug carriers ranging from 10 to $1000 \mathrm{~nm}$ in which a biologically active substance is entrapped, encapsulated or adsorbed onto the surface. 
Nanoparticles formulated from biodegradable polymers such as poly(lactic acid) (PLA) and poly(lactic-co-glycolic acids) (PLGA) were extensively investigated as non-viral gene delivery systems due to their biocompatibility $[165,166]$. PVA (poly(vinyl) alcohol)-chitosan blends can be used to stabilize the PLGA nanospheres and form a homogeneous cationic population that can bind DNA on its surface by electrostatic interactions [165]. They can be used in association with PEI to decrease particle size $[167,168]$. Nevertheless, results of in vitro transfection and cell viability on HEK 293 cells [167] and in the human bronchial cell line Calu-3 [168] exhibited weak transfection as compared to PEI alone [167].

In 1991, Bertling et al. [169] first introduced cationic polyalkylcyanoacrylate (PACA) nanoparticles as drug delivery systems for pDNA delivery, where nucleic acids were also adsorbed on the surface. Later, a large variety of cyanoacrylate polymers were associated with nucleic acids : polybutylcyanoacrylate (PBCA), polyisobutylcyanoacrylate (PIBCA) [170172], polyisohexylcyanoacrylate (PIHCA) [170, 171, 173, 174], and polyhexylcyanoacrylate (PHCA) [175]. These cationic nanoparticles allow the neutralization of the negative charge of nucleic acids, allowing an efficient interaction with cellular membranes. As an example, Chavany et al. [173] carried cell uptake studies of ODN adsorbed onto PIHCA nanospheres. They showed that the uptake of the ODNs was dramatically increased when associated with nanospheres. After $24 \mathrm{~h}$ incubation, uptake of oligonucleotide was 8-time higher when adsorbed to nanospheres than when incubated as an ODN free. Regrettably, up to now, very little is known about in vivo applications of these drug carriers.

These kinds of adsorbing systems lead to a better cellular internalization compared to DNA alone, nevertheless the fact of exposing nucleic acid adsorbed on the nanoparticle surface prevents from the use of a systemic injection since the DNA will not be protected from the hostile DNase environment, and opsonines. Consequently, for systemic gene delivery, the idea of encapsulating nucleic acids inside polymer based systems was rapidly 
developed to provide an efficient protection of nucleic acids and a long-term release in the required environment. Therefore, the development of nanocapsules containing nucleic acids in their aqueous core was considered. Nanocapsules were prepared by interfacial polymerization of isobutylcyanoacrylate (IBCA) in a W/O emulsion. The nanocapsules displayed a size of $350 \pm 100 \mathrm{~nm}$, a zeta potential of $-40 \mathrm{mV}$ and were able to encapsulate efficiently high amounts of phosphorothioate oligonucleotides (ODN) directed against EWS Fli-1 chimeric RNA (a translocation found in $90 \%$ of both Ewing's sarcoma and primitive neuroectodermal tumor). Moreover, it permitted to obtain inhibition of Ewing sarcoma-related tumor in mice after intratumoral injection of a cumulative dose as low as 14.4 nanomoles $[176,177]$.

Liu et al. [178] chose to formulate ultra low size calcium phosphate nanoparticles entrapping DNA molecules. The average diameter of the particles was approximately 23.5$34.5 \mathrm{~nm}$ and they displayed a zeta potential of $+16.8 \mathrm{mV}$. In vitro studies showed that these nanoparticles efficiently encapsulated the DNA, protected it from DNAse degradation. Assays on suicide gene therapy (the delivery of a gene able to transform a pro-drug into an active drug, injected in a second time) displayed good results. However, these nanoparticles were not tested via systemic injection.

Aynie et al. [179] developed a process which led to the preparation of spongelike alginate and polylysine nanocapsules. The resulting so-called nanosponges displayed a size of $320 \mathrm{~nm}$ and a zeta potential of $-34 \mathrm{mV}$. Alginate nanosponges lead to an important protection of AS-ODN: $80 \%$ remained undegraded after one hour incubation in fetal calf serum. 


\section{Lipid based systems}

A novel pDNA-nanoparticle delivery system (synthesized from emulsion) was developed by entrapping hydrophobized pDNA inside nanoparticles engineered from microemulsion precursors. Plasmid DNA was hydrophobized by complexing with cationic surfactants DOTAP and DDAB. Warm microemulsions were prepared at $50-55{ }^{\circ} \mathrm{C}$ with emulsifying wax, Brij ${ }^{\circledR} 78$, Tween $^{\circledR} 20$, and $\operatorname{Tween}^{\circledR} 80$. Nanoparticles were engineered by simply cooling the microemulsions containing the hydrophobized pDNA in the oil phase to room temperature while stirring. Biodistribution studies have showed a long circulation time and, 30 minutes after tail vein injection to mice, only $16 \%$ of the 'naked' pDNA remained in the circulating blood compared to over $40 \%$ of the entrapped pDNA [180]. However, no transfection studies have been carried out in vivo.

For a decade, trials have been undertaken to utilize solid lipid nanoparticles (SLN) as alternative drug delivery systems [181]. The suitability of cationically modified SLN as a novel transfection agent was investigated. Only one SLN batch composed of $4 \%$ Compritol ${ }^{\circledR}$ ATO 888 (as matrix lipid), 4\% Tween ${ }^{\circledR} 80 / \operatorname{Span}^{\circledR} 85$ (as surfactant) and 1\% EQ1 (N,N-di-(bsteaorylethyl)- $N, N$-dimethylammonium chloride) (as charge carrier), was able to form stable complexes with DNA. Typical complexes were 300 to $800 \mathrm{~nm}$ in size, with a surface charge around $+40 \mathrm{mV}$. Cytotoxicity and transfection studies exposed a good tolerance of the complexes, and an efficient transfection [181]. However, this transfection remained very low in comparison to conventional agents [182].

Taking into account the large size and the high charge of these encapsulating systems, their in vivo behaviour is uncertain. In fact, as seen previously ( $\$ 3.1$ ), size and charge are important factors influencing the activation of the immune system. Thus, for the majority of the cited encapsulating systems, biodistribution studies displayed a localization in the MPS organs and a subsequent distribution in the other organs $[171,172,179]$. These 
systems are therefore weakly efficient for the systemic delivery of nucleic acids; nevertheless it may be a useful method for local administration of a therapeutic gene, such as the pulmonary route or directly into a solid tumor.

\subsection{3 - Positive charge dissimulation}

For increasing the efficiency of gene therapy via systemic delivery, it is necessary to mask the net positive charge of these carriers to enable the circulation of complexed DNA in the blood stream. To avoid non-specific interaction with blood components, modification can be accomplished by "shielding" the vector surface with a hydrophilic and flexible polymer such as PEG. In 1990, Klibanov et al. [183] exposed for the first time that PEG use could enhance the circulation time of liposomes. After this, Campbell et al. [184], found that cationic lipids stabilized with the addition of PEG could accumulate in a tumor through its leaky vasculature according to the "enhanced permeability and retention (EPR) effect". Indeed, tumor tissues display several distinct characters such as hypervascularization, defective vascular architecture and a deficient lymphatic drainage system, which lead carriers to accumulate preferentially and to be more retained in tumor tissues than in normal tissues $[185,186]$. Today, numbers of the gene delivery systems (including lipoplexes, as well as polyplexes or nanoparticles) aimed at systemic injection are PEGylated to provide enhanced circulation time in the bloodstream [187]. This effect is the result of a high hydrophilic profile, combined with brushtype polymercrowding and flexibility [98], which prevents from opsonisation and capture by macrophages. PEG capacity for repelling proteins and not interacting with macrophage plasma membrane is largely dependent on different parameters such as the molecular weight (MW), the density, the conformation and the flexibility of chain (for review see Vonarbourg et al. [98]). Most of author advocate an efficient MW in the range 
of 1500 to $3,500 \mathrm{Da}$ for decreasing protein adsorption in vitro, but concerning the macrophage uptake, chains have to be very long to be efficient $(20,000 \mathrm{Da})[188]$. To resume, the MW and the density are important criteria that can compensate each other and are related to each another in order to create a sufficient thickness limiting interaction with proteins and/or macrophages [189]. Moreover, the PEG molecules can be modified to improve the target specificity of the PEGylated particles [190].

\section{Pegylated polymer based systems}

Numerous sort of modified PEI have been synthetized in order to increase the polyplexe circulation time within the bloodstream, and to decrease its toxicity [191-193]. Results showed that the degree of PEGylation and the molecular weight of PEG strongly influenced the properties of the resulting PEG-PEI conjugates [194]. While most of the groups grafted the PEG onto PEI first and formed the DNA complexes in a subsequent step, Wagner et al. first condensed DNA with PEI $800 \mathrm{kDa}$ and subsequently grafted on the hydrophilic polymer to provide a better condensation of the DNA with the polycations [20, 93].

The covalent coupling of PEG can be carried out via the primary amino groups of the PEI molecules by reaction with the succinimidyl derivative of PEG. Recently, the PEGylation of dendritic PLL caused great changes in enhancing blood residence and reducing hepatic accumulation [195, 196]. Another promising coated type of gene delivery system is a polyelectrolyte complex (PEC) micelle-based vector. PEC micelles are the result of interactions between PEG conjugated oligonucleic acids (PEGylated ODN) and a polycations (e.g PEI). The negatively charged antisens c-Raf ODN interacts with polycations to form a neutral charged hydrophobic inner core of PEC micelles, while PEG segments are localized outside the core to form a hydrophilic shell. Thus, compared to ODN alone, the micelles 
showed a superior antiproliferative activity against ovarian cancer cells in vitro and in vivo when injected intratumorally [197].

Gelatin, a compound currently employed in pharmaceuticals and cosmetics, as well as food products, was also used to form 200nm nanoparticles containing DNA. The amine residues of basic amino acids can be easily modified with PEG derivatives to confer longcirculating properties to the nanoparticles $[198,199]$. In vitro studies showed that these nanoparticles were internalized by non-specific endocytosis pathway and able to deliver their content in the peri-nuclear region within 12 hours. The transfection efficiency of these PEGylated gelatin nanoparticles was significantly higher than for classic gelatin nanoparticles [200]. Very recently, Kommareddy et al. [201] showed an efficient in vivo expression of a pDNA encoding for the soluble form of the extracellular domain of vascular endothelial growth factor receptor-1 (VEGF-R1 or sFlt-1) after intravenous administration in female nude mice bearing orthotopic MDA-MB-435 breast adenocarcinoma xenografts. These PEGmodified gelatin-based nanovectors are therefore promising systems for an effective systemically administered gene delivery vehicle in solid tumor.

Another important and promising example of polymer therapeutics concerns polymer micelles formed by amphiphilic block copolymers. As an example, Pluronic ${ }^{\circledR}$ block copolymers consist of ethylene oxide (EO) and propylene oxide (PO) blocks arranged in a triblock structure: $\mathrm{EO}_{x}-\mathrm{PO}_{y}-\mathrm{EO}_{\mathrm{x}}$. These amphiphilic copolymers self assemble into micelles in aqueous solution. Interestingly, in vitro, the transfection efficiency of poly( $N$-ethyl-4vinylpyridinium bromide)-pDNA complexes and asialo-oroso-mucoid-poly(L-lysine)-pDNA complexes was significantly increased in the presence of free Pluronic ${ }^{\circledR}$ F85 and Pluronic ${ }^{\circledR}$ F127, respectively $[202,203]$. In another study, a block graft copolymer synthetized by covalent conjugation of Pluronic ${ }^{\circledR}$ P123 and branched polyethyleneimine [P123-g-PEI (2K)] formed a stable complex with pDNA (110nm), and exposed higher or similar gene expression 
than well-known lipid transfection reagents (Lipofectin ${ }^{\circledR}$, Lipofectamine ${ }^{T M}$, CeLLFECTIN $^{\circledR}$ and DMRI-C) in the spleen, the lung, the heart and the liver $24 \mathrm{~h}$ after i.v injection and produced relatively low toxicity [204]. As well as pDNA encapsulation, a system of P85-g-PEI(2K) was used for preparing AS-ODN complexes and these also showed their accumulation almost exclusively in the liver, predominantly in hepatocytes, whereas only a small amount was found in the lymphocytes/monocytes population [205]. The high stability is due to the formation of micelle-like structures: electrostatic binding of the nucleic acids and PEI chains of the P123-g-PEI (2K) or P85-g-PEI (2K) results in the neutralization of positives charges of the cationic polymer PEI and as a result, the formed particles bear a surface charge close to zero. Therefore, it is likely that these systems were stabilized in dispersion by the EO corona in a manner similar to classic Pluronic ${ }^{\circledR}$ micelles. Moreover, the PO chains in pluronic molecules are known to interact with lipidic membranes and induce their structural rearrangement. Contrary to EO chains, the presence of PO chains allows the carriers to translocate the carriers. Pluronic ${ }^{\circledR}$ based polyplexes, such as P123-g-PEI (2K) graft block polymer complexes, are promising vectors for gene delivery which have many advantages compared to 'simple' polyplexes (such as high stability in dispersion and high transfection activity).

PEG is currently the most used polymer in coating methods, but other hydrophilic polymers can be employed. Bharali et al. [206] synthesized poly(vinylpyrrolidone) (PVP) nanoparticles and demonstrated that these particles on i.v administration could evade the MPS and remain in circulation for a considerable period of time. This hydrophilic polymer is known to be biocompatible, non-antigenic and is therefore safe for animal experiments [206]. DNA could be successfully encapsulated in PVP nanoparticles and could be protected from nucleases. The reporter gene $\mathrm{PSV} \beta$-gal was encapsulated and the in vitro transfection efficiency of this system was found to be nearly $80 \%$ compared to the highly-successful in 
vitro transfection reagent Polyfect ${ }^{\circledR}$ (derived from dendrimers). Further in vivo biodistribution studies have indicated that this system could be used safely for effective gene delivery [207].

\section{Pegylated lipid based systems}

Stabilized plasmid-lipid particles (SPLP) are systems associating small size (100 +/$10 \mathrm{~nm})$ and encapsulation. They are synthesized by a detergent dialysis procedure and consist of a unilamellar lipid bilayer containing (usually) the cationic lipid DODAC (N,Ndioleyl-N,N-dimethylammonium chloride), the neutral helper lipid DOPE, and PEG conjugated ceramides (PEG-Cer), surrounding a single copy of plasmid DNA [208]. Nevertheless, low transfection activity was observed.

Moreover, although SPLP showed potential for systemic gene transfer, the detergent dialysis method of preparation suffered from a number of limitations making any clinical application impossible [209]. A fully scalable and extrusion-free method (by spontaneous vesicle formation) was developed to rapidly prepare reproducible stabilized plasmid lipid particles. Additionally, this method accelerated SPLP formulation, enabling the rapid development and evaluation of novel carrier systems, also called stabilized nucleic acid lipid particles (SNALP) when encapsulating siRNA. Using these sytems, Zimmermann et al. [210] reported for the first time an siRNA-mediated silencing of the apolipoproteinB $(A p o B)$ in response to systemic delivery in non-rodent species. In this study, SNALP were composed of lipids 3- $N$-[(-methoxypoly(ethyleneglycol) 2000$)$ carbamoyl]-1,2-dimyristyloxy-propylamine (PEG-C-DMA), 1,2-dilinoleyloxy-N,N-dimethyl-3-aminopropane (DLinDMA), 1,2-distearoyl-snglycero-3-phosphocholine (DSPC) and cholesterol, in a 2:40:10:48 molar per cent ratio. SNALP containing ApoB-specific siRNAs were administered by intravenous injection to cynomolgus monkeys at doses of 1 or $2.5 \mathrm{mg} \mathrm{kg}^{-1}$. Significant reductions in ApoB protein, serum cholesterol and low-density lipoprotein levels were observed as early as $24 \mathrm{~h}$ after 
treatment and lasted for 11 days at the highest siRNA dose, thus demonstrating an immediate, potent and lasting biological effect of siRNA treatment. This study exposed for the first time a clinically relevant systemic RNAi-mediated gene silencing in non-human primates [210].

A novel cationic lipid AtuFECT01 (b-L-arginyl-2,3-Ldiaminopropionic acid-N-palmityl-Noleyl-amide trihydrochloride, Atugen AG), the neutral phospholipid 1,2-diphytanoyl-snglycero-3-phosphoethanolamine (DPhyPE) (as fusogenic lipid) and the PEGylated lipid N(carbonyl methoxypolyethyleneglycol-2000)-1,2-distearoyl-sn-glycero-3-phosphoethanolamine sodium salt (DSPE-PEG) were mixed in a molar ratio of $50 / 49 / 1$ to form a complex with siRNA named AtuPLEX (with a size of $117.8 \mathrm{~nm}$ and a surface charge of 46.4 $\mathrm{mV}$ ). By using siRNA molecules for targeting endothelia-specific expressed genes, such as CD31 and Tie2, Santel et al. [211] demonstrated downregulation of the corresponding mRNA and protein in vivo after repeated systemic i.v. administration ( 1 / day for 4 days) in mice [211] [212].

Other particle models were synthesized, such as the stabilized antisens-lipid particles (SALP), that have characteristics close to SPLP ones, but are constituted of multilamellar vesicles resulting in an ionizable aminolipid (1,2-dioleoyl-3-dimethylammonium propane, DODAP) and an ethanol-containing buffer system for encapsulating large quantities (0.15$0.25 \mathrm{~g}$ ODN / g lipid) of polyanionic ODN [213]. In the same way, Pagnan et al. [214] chose to formulate coated cationic liposomes (CCL) composed of (hydrogenated soy phosphatidylcholine (HSPC), cholesterol, DSPE-PEG 2000, and DOTAP. These systems resulted from the shielding of cationic lipid/DNA complexes by a neutral liposomal membrane where PEG residues are inserted. This CCL associated with monoclonal antibodies targeting disialoganglioside GD2, extensively expressed in neuroectodermal tumors, can encapsulate 
AS-ODN against c-myc proto-oncogene and displays interesting anti-tumor effects in vivo [215].

\subsection{4 - Solutions to the limitations of PEG coating}

On one hand, the PEG coating increases blood circulation time, which consequently leaves time for the objects to reach tumors thanks to the EPR effect $[20,27,216,217]$. But on the other hand, the PEG shield represents a major barrier for internalization and endosomal escape [218-220].

\section{Use of removable PEG}

One solution is to use a dynamic, environment-responsive, movable PEG as exploited by one of the most promising vector, the SPLP. It has been established that the in vitro transfection potency of SPLP is dependent on the hydrophobic Cer group anchoring the PEG polymer to the SPLP, where Cer groups, containing shorter acyl groups, exhibited improved transfection properties [208].This is attributed to the ability of PEG-Cer molecules with shorter acyl groups to dissociate from the SPLP, thereby destabilizing the particle and improving association with and uptake into target cells [221]. The transfection levels achieved for SPLP containing PEG-CerC 8 were substantially higher than for SPLP containing PEG-CerC ${ }_{14}$ or PEG-CerC 20 , this being consistent with the necessity for the PEG-Cer to dissociate itself from the SPLP surface for maximum transfection efficiency [222]. It should be noted that the SPLP were recently prepared using a series of PEG diacylglycerol lipids (PEG-S-DAG). These constructs are more easily synthesized and exhibited extended circulation lifetimes and tumor-selective gene expression compared to SPLP containing the PEG-Cer [223]. This kind of SPLP (PEG-S-DAG or PEG-Cer) has been shown to bypass the so-called 'first pass' 
organs (including the lung, liver and spleen) and elicit levels of gene expression in distal tumor tissues 100 - to 1,000 -fold greater than that observed in normal tissues. Furthermore, some improvements in transfection were noted when short PEG polymers (PEG 750) were incorporated into the PEG-Cer rather than PEG 2000 or PEG 5000 polymers [222]. SPLP systems are an example of the ambiguous role of PEG coating. A compromise has to be found between a sufficient circulation time in the bloodstream and good transfection efficiency [224].

Additional to the internalization problems engendered, PEG coating could also impede the endosomal escape of the gene-delivery system. To resolve this problem, the neutralizing PEG shield could be attached to the polyplexe core via an acid labile linkage that, confronted with the acidic environment of the endosome compartment, will be hydrolyzed, providing the shielding destabilization. Chemical linkages that may display $\mathrm{pH}$-dependent hydrolytic degradation once internalized into endosomal and lysosomal compartments include acetals [225, 226], vinyl ether [227], ortho esters [228] and hydrazones [229].

To improve internalization and endosomal escape, Walker et al. [230] chose to link PEG to PLL and PEI via a pH-sensitive hydrazone bond. The targeting ligand transferrin was also included to the polyplexes. Their in vitro and in vivo studies showed that receptortargeted polyplexes generated with this kind of PEG led to dramatically higher transfection efficiency in targeted cells compared to those generated with stable shielded control polyplexes. In the same way, Choi et al. [228] chose to use an acid labile PEG-diorthoesterdistearoylglycerol lipid (POD) mixed with a cationic lipid (DOTAP) and phosphatidylethanolamine (PE) to prepare SPLP (POD-SPLP) that could mediate in vitro gene transfer by a pH-triggered escape from the endosome. This POD-SPLP exposed up to 3 times greater gene transfer activity in vitro than $\mathrm{pH}$-insensitive nanoparticle. Moreover, both the $\mathrm{pH}$-sensitive and $\mathrm{pH}$-insensitive nanoparticles were internalized to a qualitatively similar 
extent, underlining that increased gene transfer of the POD-SPLP was due to a faster escape from the endosome rather than to greater cell association of these nanoparticles [228].

\section{Cell-specific targeting, ligand attachment}

To avoid the problem of non-specific interaction and to overcome the difficulties encountered with PEG coating, a target-specific ligand can be added to the gene-delivery system (Table 2) resulting in active targeting and receptor-mediated endocytosis [218, 231, 232]. Gene-delivery vectors should allow specific cell types to be targeted by utilizing the interactions between cell surface receptors and ligands present on their surface. These ligands can be small molecules (e.g. folate, galactose, low-density lipoprotein (LDL), fibroblast growth factor (FGF) receptor binding peptides,.ect) [196, 233-235] or peptides and proteins (e.g. transferrin or antibodies). Numerous studies have been carried out. For example, transferrin is a common ligand used to target tumor cells [236, 237]. Repeated systemic application of surface shielded transferrin-polyethyleneimine (Tf-PEI)/DNA delivery systems promoting the expression of the tumor necrosis factor (TNF)- $\alpha$ (carrying immunostimulatory and cytotoxic properties) into tumor- bearing mice induced tumor necrosis and inhibition of tumor growth in four murine tumor models [238]. Because the expression of TNF- $\alpha$ was largely localized within the tumor, no significant systemic TNF-related toxicities were observed. In the same way, $\mathrm{Xu}$ et al. [239] chose to incorporate an anti-human transferrin receptor single-chain antibody Fv fragment (TfRscFv) into the lipoplexes (DOPTAP/DOPE/DOPC) as an alternative targeting ligand. More recently, they developed an improved formulation with a sequential conjugation of lipoplexes with PEG and TfRscFv, and demonstrated enhanced expression in vivo of genes encoding p53 and green fluorescent protein (GFP) in prostate tumors [240]. 
With the goal of targeting tumor vasculature, RGD peptides (specifically targeting specifically the up-regulated integrin receptors in certain tumors) were attached to a pegylated PEI system containing siRNA against vascular endothelial growth factor (VEGF) receptor-2 [241]. Intravenous administration into tumor-bearing mice resulted in selective tumor uptake, siRNA sequence-specific inhibition of protein expression within the tumor and the inhibition of both tumor angiogenesis and growth rate.

Lipoplexes grafted with galactose (Gal-lipoplexes) were used to target hepatocytes through specific recognition by the asialoglycoprotein receptor and to reduce non-specific uptake by Kupffer cells [242, 243]. In the same way, Reddy [244] and Hofland [245] chosen to use folate to target folate receptor known to be overexpressed in a large variety of human tumors and basically expressed in normal tissues. They succeeded in developing folateconjugated lipoplexes that exposed in vivo transgene expression in mice tumors.

Epidermal growth factor (EGF)-receptor expression is often increased in breast or prostate cancers, making it a good candidate for targeting gene-transfer complexes. Blessing et al. [246] used an EGF-targeted PEl-based system and obtained highly increased transgene expression in vitro. ErbB2, a tumor marker that is highly upregulated in many human breast and prostate cancers, was targeted with a delivery system (protamine and cationic lipids) containing a single-chain antibody [247]. It should be noted that when using antibody, the lack of the Fc-fragment of the antibody can avoid recognition by macrophages and thus clearance from the bloodstream. In order to target the tumor specific marker PSMA (prostate-specific membrane antigen), an anti-PSMA monoclonal antibody, J591, was generated and showed cellular internalization. Therefore, Moffat et al. [248] envisaged that a J591/PEI/DNAß-gal vector could be harnessed in order to target PSMA in the prostate tumor. The in vivo organ distribution profile revealed gene expression predominantly in the tumor. Whatever the choice of ligand, it seems important to place it several nanometers away from 
the surface of the particle to provide an effective binding to cell-surface receptors. Several investigators have already applied this concept, and projected the targeting ligand away from polyplexes through PEG linkers, where the ligand is attached at the distal end of the PEG [236, 246, 249].

As seen before (§4.1.1), 'naked' LPD nanoparticles were developed for pDNA delivery, but these carriers tends to aggregate in the presence of serum protein due to their high surface charge [147]. Recently, Li et al. [250] formulated AS-ODN and siRNA in LPD, and further introduced PEGylated lipid by the postinsertion method to induce serum stabilization. Anisamide, a compound that specifically binds to sigma receptor (over-expressed in human lung cancer cells) was added to the distal end of PEG for tumor targeting. In vitro studies exposed that this ligand increased the delivery efficiency by 4-7 fold for sigma receptor overexpressed cells. Therefore, the PEGylated, anisamide-targeted LPD showed a strong potential to deliver systemically oligonucleotides for cancer therapy.

Recently, Green et al. [251] linked their ligand by electrostatic interactions with cationic polymeric gene-delivery nanoparticles. This kind of RGD coated nanoparticle enabled effective ligand-specific gene delivery to human primary endothelial cells in serum-containing media, suggesting promising results for an in vivo application.

To further increase specificity and safety of gene therapy, the expression of therapeutic genes can be tightly controlled within the targeted tissue. The presence of tissue or environment-specific promoter can allow the activation of gene expression in diseased states, or in unfavourable tumor-associated microenvironment, for example, hypoxia (for review see [252]).

\section{2 - Intracellular trafficking}




\subsection{1 - Internalization}

With the goal of improving transfection efficiency, the use of protein transduction domain (PTD) has been tested to mediate an endocytosis-independent cellular uptake of proteins as well as large molecules [253]. Several sequences exist that have been identified as possessing transducing efficiency, among them the TAT protein derived from the HIV-1 virus [254]. As proof of the concept, the SLN transfection efficiency was significantly improved in vitro by pre-complexing DNA with a dimer of the HIV-1 TAT-peptide, which contains a cell-penetrating domain for the improvement of cellular uptake and a nuclear localization sequence for the translocation of DNA into the nucleus [255]. It was demonstrated that liposomes $(200 \mathrm{~nm})$ injected at the tumor site can be delivered directly into cytoplasm without causing major damage of the vesicles by attaching TAT peptides to their surface via a PEG spacer in order to decrease steric interaction [256]. This study showed that the attachment of TAT peptides led to significantly higher transducing efficiency. It can be noted that the TAT peptide, because of its viral origin, still has the risk of being recognized by the immune system in the case of a systemic injection.

The knowledge of how PTDs mediate cell entry is currently discussed. Until recently, it was widely assumed that the internalization of cationic PTDs was an energy and receptorindependent process based on direct transport through the lipid bilayer [257-259]. On the other hand, there have been indications that the uptake of full-length TAT protein, occurs via endocytosis and depends on cell surface heparan sulfate receptors [260]. Recently, numerous possible internalization routes for TAT have been proposed, such as lipid-raft mediated macropinocytosis [261], caveolae-mediated endocytosis [262, 263] and clathrindependent endocytosis [264]. However, the uptake characteristics of the TAT peptide alone and of TAT-conjugated cargoes have been demonstrated to differ significantly [265-267]. Furthermore, the TAT-mediated internalization process has been proposed as being 
dependent on the properties of the cargo molecule, TAT concentration and the cell line [265, 268]. To conclude, the mechanism of PTD-mediated delivery remains elusive, and may depend on the vector associated with PTDs.

A new concept for the development of vectors is to overcome the limitations of individual vectors by combining them. In this way, virosomes were synthesized, resulting in a mix between viral glycoprotein and anionic liposomes, in which DNA is encapsulated under a protein complex form to allow better intracellular trafficking and nuclear entry. One example concerns the HVJ (hemagglutinating virus of Japan) liposome (or fusogenic liposome) which is composed of HJV glycoproteins. Indeed, some proteins of this virus possess fusogenic properties which facilitate cellular uptake by interacting with sialic residues of the cellular membrane. Such systems had efficient transfection activity in vitro and in vivo following intratissular injection $[269,270]$. Recently, to facilitate the usefulness of the HVJ-liposome for hepatic gene therapy, Kaneda's group evaluated the efficacy of the total vascular exclusion technique (TVE) during the portal vein injection (PVI) of the gene transfer vector regarding its transfection efficiency in rat liver [271]. This consists of a surgical procedure in which the liver is isolated from the surrounded tissues; HVJ-liposome solution was then infused selectively into the liver through an inserted catheter. The results indicated that PVI + TVE might facilitate the liver-specific gene delivery using the HVJ-liposome method, avoiding the extrahepatic transgene expression. Nevertheless, this technique requires a surgical handling as opposed to a 'simple' systemic injection, and this seems to be a limitation for a clinical use.

In the same way, combinatorial nanotechnology using fusogenic liposomes (having a Sendaï virus envelope, glycoprotein on the surface) encapsulating poly(vinyl amine) nanoparticles seems to be a valuable system for regulating the intracellular pharmakocinetics of gene-based drugs [272]. This class of construct is able to efficiently deliver the 
encapsulated contents to the cytoplasm through its direct fusion with the cytoplasm membrane.

This kind of combining systems could be the beginning of the promising "virus like vector" that will be able to mimic the efficient transfection of viruses. But, these systems have to be improved for in vivo application, particularly as far as immune recognition is concerned.

\subsection{2 - Endosomal escape}

With the objective of enhancing endosomal escape, $\mathrm{pH}$-sensitive endosomolytic peptide can be attached to polyplexes or lipoplexes. Viruses have developed clever mechanisms to overcome the endosomal barrier: viral proteins often contain membraneactive domains mediating the delivery of the viral genome to the cytoplasm after their activation in the endosome (for review see Ref. [273]). Among these virus peptides, we can find peptides derived from the N-terminal domain of Haemophilus influenzae haemagglutinin2 peptide [274], or synthetic peptides such as GALA [275], or KALA [276]. As a proof of the concept, fusogenic peptides were incorporated in chitosan/plasmid complex which significantly increased transfection efficiency [277]. Also, other naturally-occurring peptides and small proteins, such as those present in the venom of vertebrates and invertebrates, can be highly membrane-destabilizing (for review see Ref. [278]). Both types of compounds were already used to enhance the intracellular delivery of transferred DNA, such as influenzaderived peptides [279] or melittin (derived from bee venom) [280] which were applied in vivo to facilitate the delivery of double stranded RNA to glioblastoma [281]. However, melittin also showed pronounced lytic activity at neutral $\mathrm{pH}$, which is undesirable and responsible for toxic side effects. To overcome this limitation, Rozema et al. [282] chose to modify the lysines of melittin with a dimethylmaleic anhydride derivative, which can mask the lytic activity at neutral 
$\mathrm{pH}$. Concerning endosomal acidification, the masking groups are removed and the lytic activity of melittin is restored. Other approaches to $\mathrm{pH}$-dependent lytic activity include acidic melittin analogs [283] or the incorporation of melittin into bioreducible copolymers [284].

An original concept was proposed to disrupt endosome and lysosome membranes in a desired location (e.g tumor), called photochemical internalization (PCl). Indeed, the cytoplasmic delivery of macromolecular compounds can be enhanced by the photochemical disruption of the endosomal membrane using light and a hydrophilic photosensitizer. This smart concept is, in principle, applicable to in vivo gene delivery in a light-sensitive manner [285]. However, the cytotoxicity is due to photochemical reactions in the cell, and this might need to be reduced before considering further applications of this technology. If not, damage to organelles other than the endosomal membranes, for example plasma or mitochondria membranes could occur. Nishiyama et al. [286] chose to develop a light-responsive carrier based on a ternary complex of pDNA, cationic peptides, and anionic dendrimer-based photosensitizer (dendrimer phtalocyanine). This sophisticated system seems to reduce the toxicity and to enhance the efficiency of transfection, leading to a 100 -fold improvement in the level of transgene expression using light irradiation. Polyplexes consisting of pDNA and glycosylated PEI were used for in vivo p53 gene transfer in mice bearing head and neck squamous cell carcinoma xenografts. The treatment was combined with $\mathrm{PCl}$ technology. $\mathrm{PCl}$ led to a 20 -fold increase of sustained transgene expression. Weekly treatment repeated for 7 weeks resulted in tumor growth inhibition in all animals and cured $83 \%$ of the mouse population [287].

4.2.3 - Nucleus entry 
Interestingly, viral particles can travel through the cytoplasm by interaction with the micro-tubular network and have various mechanisms for traversing the nuclear membrane. The observation that linear PEI seemed to assist these transport processes, whereas branched PEI did not, was of interest [143, 288].

The NLS concept has been used to improve the uptake of plasmid DNA into the nucleus. Several groups demonstrated that the covalent attachment of an SV40 nuclear localization sequence (SV40NLS) either directly to DNA or to polymers that form complexes with DNA led to an increase of nuclear import resulting in enhanced protein expression [289291]. Branden et al. [292] demonstrated that a PNA (peptide nucleic acid) molecule linked to an SV40 NLS peptide can work as a nuclear targeting signal when hybridized to a fluorescence-labeled oligonucleotide or to a plasmid. Similar results were obtained using DOTAP or $25 \mathrm{kDa}$ PEl as transfection reagents in HeLa, NIH-3T3, or Cos-7 cells [292].

Recently, a bifunctional targeting vector was made, consisting of a recombinant reovirus type $3 \sigma 1$ attachment protein modified with a SV40-derived NLS covalently attached to the PEI to create an efficient DNA-delivery vehicle. This allowed these carriers to specifically bind to plasma membrane cell surface receptors when located outside the cell and engage the nuclear import machinery for enhanced nuclear translocation after uptake into cells. Currently, pDNA complexed with the PEI-o1-NLS delivery vehicle resulted in substantially greater levels of in vitro gene expression [293].

Moffat et al. [294] newly synthesized a multifunctional PEI based polyplexe for systemic p53-mediated gene therapy. This pegylated vector attached with a CNGRC peptide for CD13 targeting in tumors also carry two systems targeting the nucleus: a Simian Virus (SV) 40 peptide-(nuclear localization signal)) and an oligonucleotide based nuclear signal (DNA nuclear targeting signal). This promising vector exposed a significant tumor regression and $95 \%$ animal survival after 60 days. 


\section{5 - Conclusions}

Incontestably, recombinant viral vectors are still the most efficient systems for gene transfer in comparison to lipoplexes or polyplexes [295, 296]. Although considerable improvement has been made over the last few years, the standard requirements for clinical use have not been reached in terms of efficiency and specificity, in particular via systemic injections. Gene delivery is a multi-step process that needs a multi-functional carrier to go through each step. For this reason, research should focus on the synthesis of a vector, attached with different ligands to mimic a virus: this system could be called the "artificial virus" and each component would be able to help the vector overcome the various barriers it could meet. All these improvements should decrease the toxicity of the initial cationic complexes, but can however modify the interactions with biological systems. It is thus necessary to carry on meticulous biocompatibility studies for each new system in each application.

Finally, it seems clear that the expression of a single transgene is unlikely to be sufficient to eradicate a tumor, in particular when it is diagnosed late in disease progression. Hence, multimodality therapy, including conventional therapy (surgery, chemotherapy and radiotherapy) with one or more transgenes will have to be considered to provide a "chance" of success. 


\section{Figure captions}

Figure 1: Structures of current cationic polymers used in gene therapy.

Figure 2: Structure of current cationic lipids used in gene therapy and the helper lipid DOPE

Figure 3: Schematic representation of lamellar or inverted hexagonal phase structure in the formation of lipid-DNA complexes (lipoplexes).

Figure 4: Shematic representation of the different hurdlles encountered by a gene delivery system to enter and traffic into a tumor cell.

Figure 5: Hypothesis of endosomal escape of lipoplexes and polyplexes gene delivery systems.

Figure 6: Shematic nuclear entry mechanism through Nuclear Pore Complexes (NPC).

\section{Table captions}

Table 1: Physicochemical characteristics of current cationic systems

Table 2: Example of promising systems for systemic gene delivery 


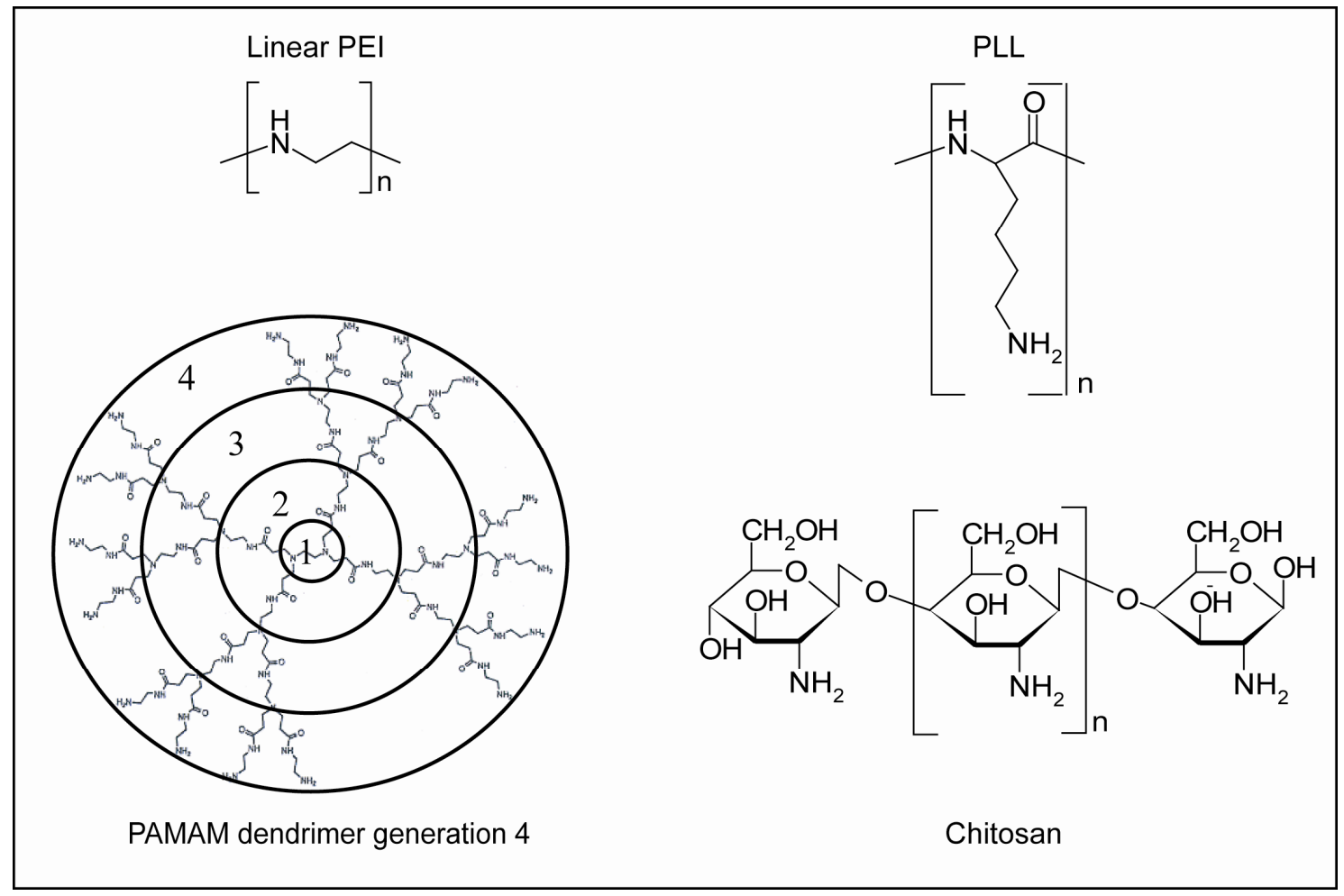

Figure 1: Structures of current cationic polymers used in gene therapy.

$\mathrm{PEI}=$ poly(ethyleneimine)

$\mathrm{PLL}=$ poly $(\mathrm{L}-$-Lysine $)$

PAMAM = poly(amidoamine) 


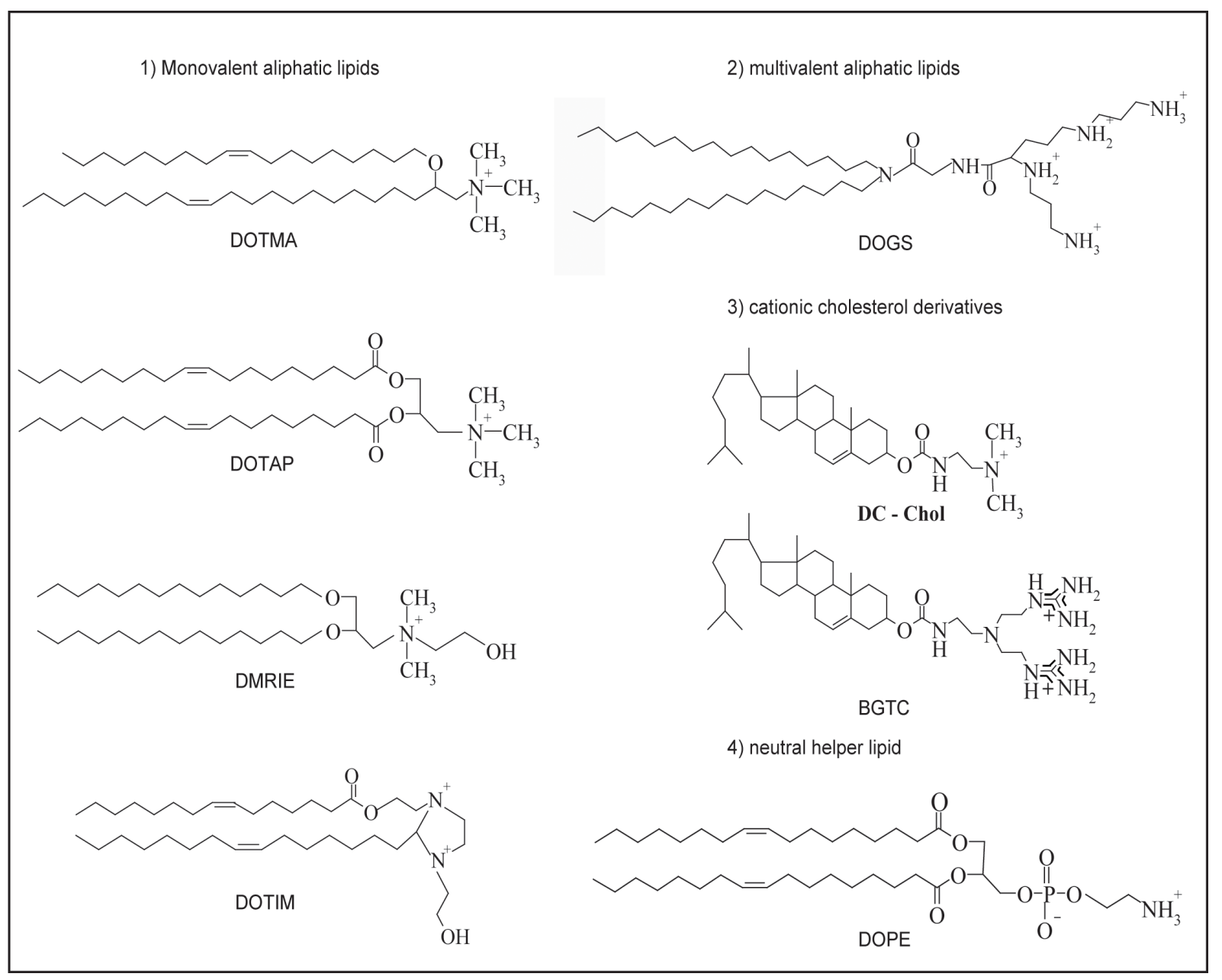

Figure 2: Structure of current cationic lipids used in gene therapy and the helper lipid DOPE. DOTMA: N [1-(2,3-dioleyloxy) propyl]-N,N,N-trymethylamonium chloride, DOTAP: 1,2-dioleyl-3-trimethylamonium-propane, DMRIE: N-(2-hydroxyethyl)-N,N-dimethyl-2,3-bis(tetradecyloxy-1-propananium bromide), DOTIM : 1-[2-(oleyloxy)ethyl]-2-oleyl-3-(2-hydroxyethyl)imidazolinium chloride DOGS: dioctadecylamidoglycylspermine DC-Chol: [N-(N',N'-dimethylaminoethane)-carbamoyl] cholesterol BGTC: bis-guanidium-tren-cholesterol DOPE: 1,2-dyoleyl-sn-glycerol-3-phosphoethanolamine 


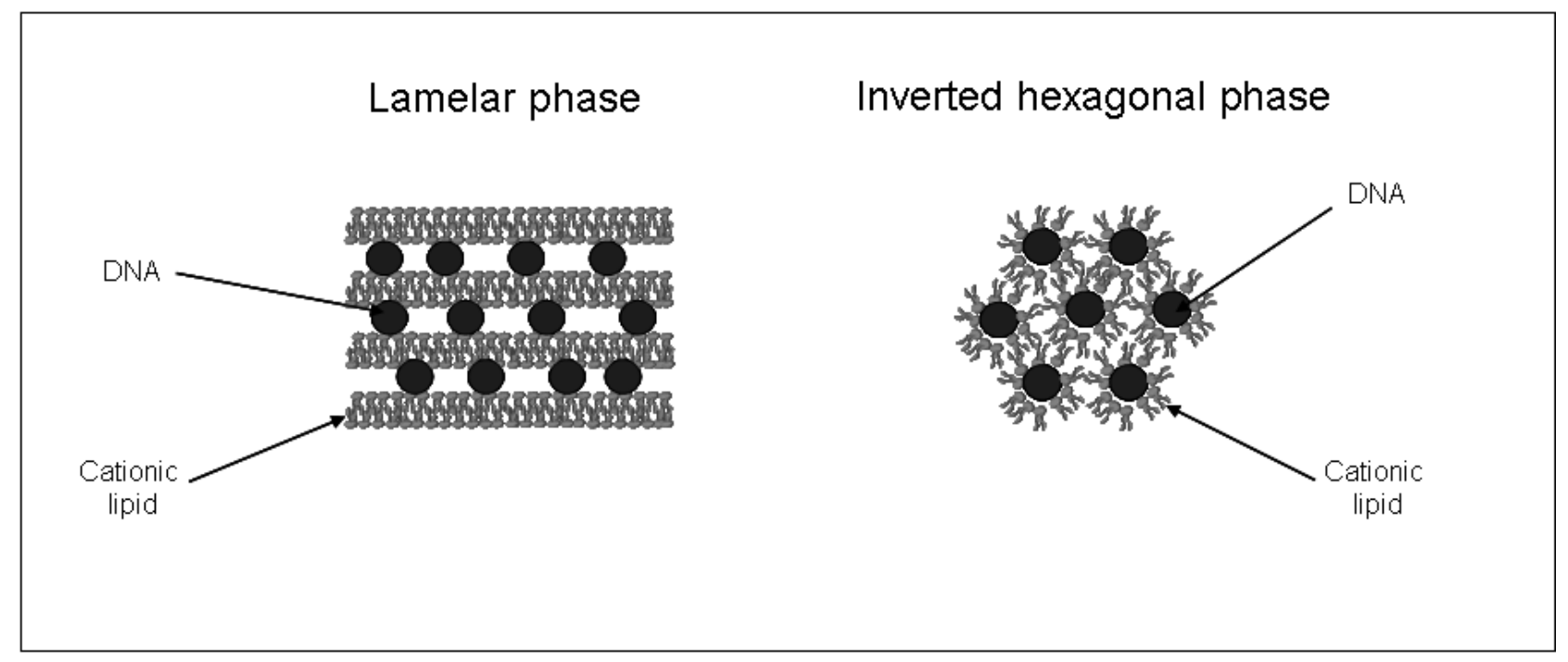

Figure 3: Schematic representation of lamellar or inverted hexagonal phase structure in the formation of lipid-DNA complexes (lipoplexes). 


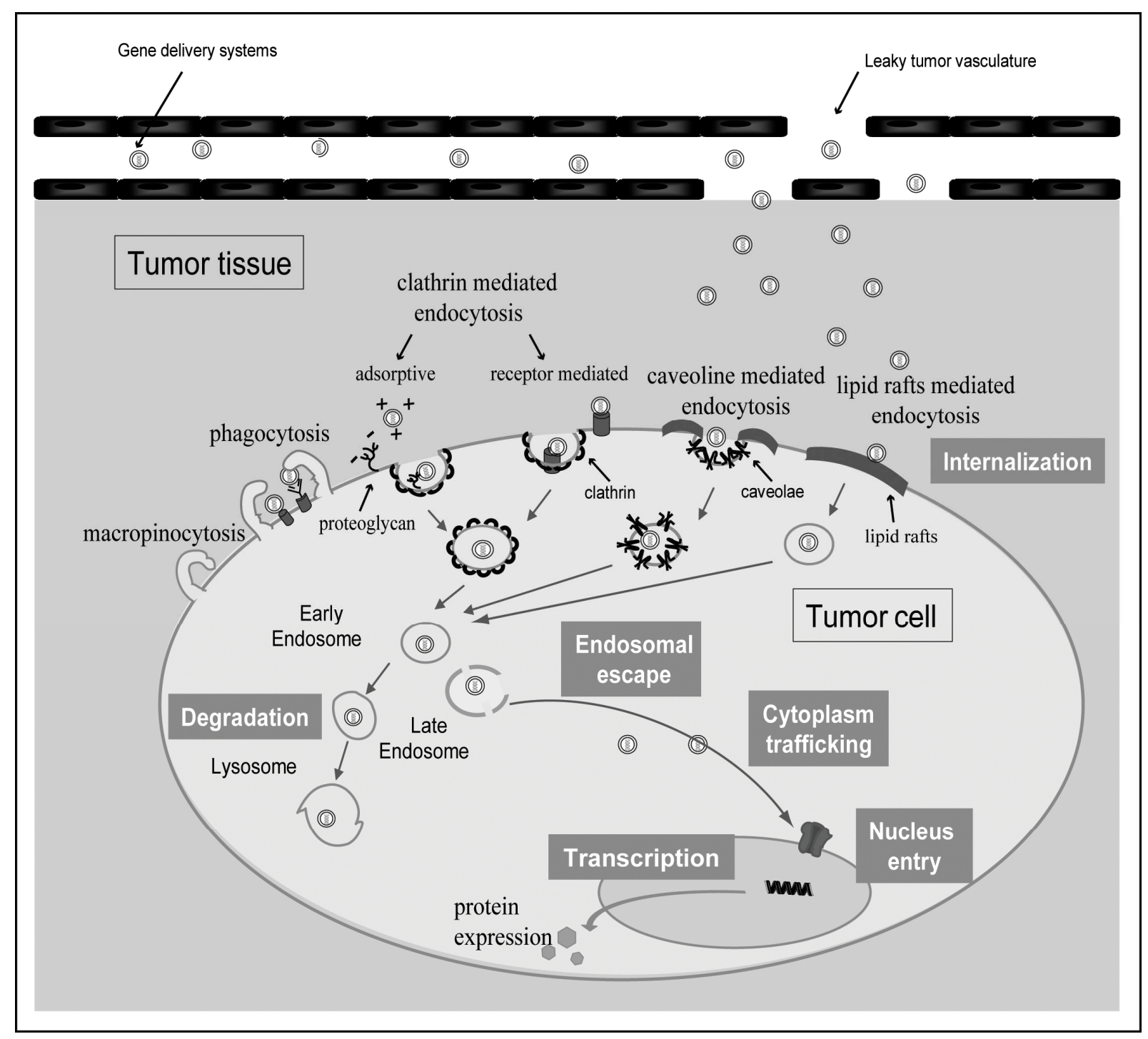

Figure 4: Schematic representation of the different hurdles encountered by a gene delivery system to enter and traffic into a tumor cell. 


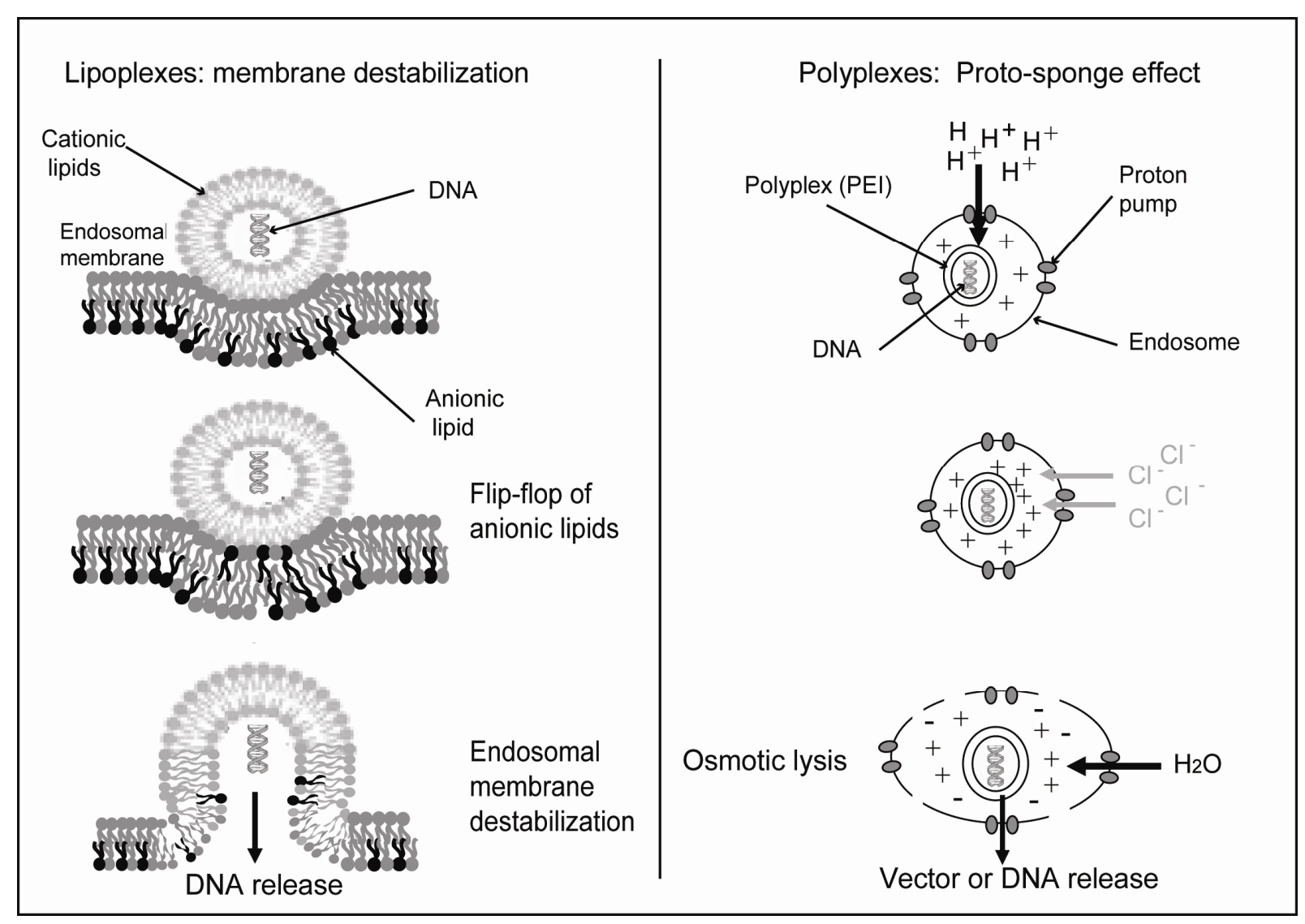

Figure 5: Hypothesis of endosomal escape of lipoplexes and polyplexes gene delivery systems. 


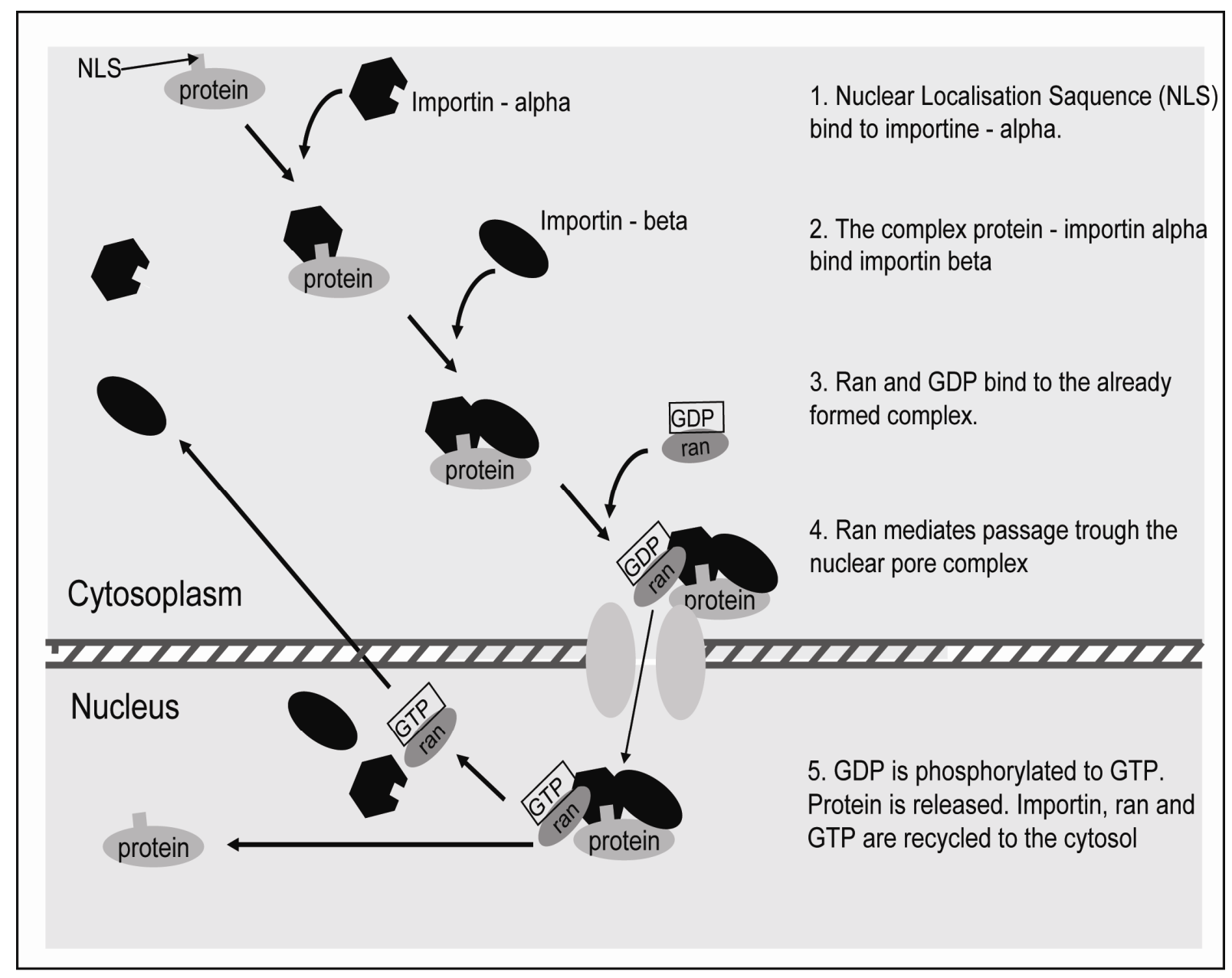

Figure 6: Schematic nuclear entry mechanism through Nuclear Pore Complexes (NPC). 


\begin{tabular}{|c|c|c|c|c|}
\hline Cationic systems * & $\begin{array}{l}\text { Particle } \\
\text { Charge }^{* *}\end{array}$ & $\begin{array}{c}\text { Particle size } \\
\text { Range of diameter }\end{array}$ & Degradability & References \\
\hline PEI - DNA & $+30 \mathrm{mV}$ & 20 to $130 \mathrm{~nm}$ & No & {$[20-21]$} \\
\hline PLL- DNA & $+40 \mathrm{mV}$ & 60 to $140 \mathrm{~nm}$ & Yes & [25-28] \\
\hline Chitosan - DNA & +25 to $+37 \mathrm{mV}$ & $20-500 \mathrm{~nm}$ & Yes & {$[34,37-42]$} \\
\hline $\begin{array}{l}\text { PAMAM dendrimer - } \\
\text { DNA }\end{array}$ & +9 to $+20 \mathrm{mV}$ & $\begin{array}{c}\text { 50-100 nm (generation } \\
6-7)\end{array}$ & Yes & {$[45-47]$} \\
\hline DOPE/DOTAP & +30 to $+50 \mathrm{mV}$ & $60-120 n m$ & Yes & [60] \\
\hline DOPE/DC-Chol & +20 to $+50 \mathrm{mV}$ & $70-120 n m$ & Yes & [60] \\
\hline DOTAP/Chol & +50 to $+60 \mathrm{mV}$ & $100-126 n m$ & Yes & [76] \\
\hline
\end{tabular}

Table 1: Physicochemical characteristics of current cationic systems

* $\mathrm{PEI}=$ poly(ethylenimine $), \mathrm{PLL}=$ poly $(\mathrm{L}$-Lysine $), \mathrm{PAMAM}=$ poly (amidoamine $)$,

$\mathrm{DOPE}=1$,2-dyoleyl-sn-glycerol-3-phosphoethanolamine, DOTAP = 1,2-dioleyl-3-trimethylamoniumpropane, DC-Chol [N-(N',N'-dimethylaminoethane)-carbamoyl] cholesterol, Chol = cholesterol.

** Depending on the $+/$ - charge ratio

*** Depending on the method of preparatio 


\begin{tabular}{|c|c|c|c|c|c|c|}
\hline Systems & $\begin{array}{c}\text { Particles } \\
\text { Size }^{\star}\end{array}$ & $\begin{array}{l}\text { Particles } \\
\text { Charge }^{\star *}\end{array}$ & $\begin{array}{l}\text { Coating } \\
\text { PEG }\end{array}$ & $\begin{array}{l}\text { Targeting } \\
\text { Ligands }\end{array}$ & Applications $^{\star \star \star}$ & Réf \\
\hline \multicolumn{7}{|c|}{ Condensing systems } \\
\hline Lipoplexes-protamine- DNA (LPD) & $98-144$ nm & $+25 \mathrm{mV}$ & $x$ & $x$ & $\begin{array}{l}\text { E1A protein } \\
\text { (in vitro) }\end{array}$ & [147-149] \\
\hline Lipoplexes-mu-DNA (LMD) & $90-150$ nm & Not informed & $x$ & $x$ & $\begin{array}{c}\text { Reporter gene } \\
\text { (in vivo: local lung application) }\end{array}$ & {$[150,151]$} \\
\hline \multicolumn{7}{|c|}{ Encapsulating systems } \\
\hline PIBCA nanoparticles & $250-450 \mathrm{~nm}$ & $-40 \mathrm{mv}$ & $x$ & $x$ & $\begin{array}{l}\text { AsODN against EWS Fli-1 } \\
\text { (in vivo : intratumorale injection) }\end{array}$ & {$[176,177]$} \\
\hline Calcium phosphate nanoparticles & $23-34 \mathrm{~nm}$ & $+16 \mathrm{mV}$ & $x$ & $x$ & $\begin{array}{l}\text { Chimeric suicide gene yCDglyTK } \\
\text { (in vitro) }\end{array}$ & [178] \\
\hline Spongelike alginate nanoparticles & $320 \mathrm{~nm}$ & $-34 \mathrm{mV}$ & $x$ & $x$ & $\begin{array}{c}\text { Radiolabeled ODN } \\
\text { (in vivo : systemic injection) }\end{array}$ & [179] \\
\hline Solid lipid nanoparticles (SLN) & $300-800 \mathrm{~nm}$ & $+40 \mathrm{mV}$ & $x$ & $x$ & Reporter gene (In vitro) & [181-182] \\
\hline Cationic copolymer (P123-g-PEI)-DNA complexes & $110 \mathrm{~nm}$ & Close to zero & $\begin{array}{l}\text { PEO moiety of P123 } \\
\text { blocks copolymer }\end{array}$ & $x$ & $\begin{array}{c}\text { Reporter gene } \\
\text { (In vivo : systemic injection) }\end{array}$ & [204] \\
\hline Stabilized plasmid lipid particles (SPLP) & $90-110 \mathrm{~nm}$ & Not informed & PEG-Cer or PEG-DAG & $x$ & $\begin{array}{c}\text { Reporter gene } \\
\text { (In vivo : systemic injection) }\end{array}$ & $\begin{array}{c}{[208,221-} \\
224,228]\end{array}$ \\
\hline Stable nucleic acids lipid particles (SNALP) & $129-153 \mathrm{~nm}$ & Not informed & PEG-C-DMA & $x$ & $\begin{array}{l}\text { siRNA against ApoB mRNA } \\
\text { (In vivo : systemic injection) }\end{array}$ & [210] \\
\hline Stabilized antisense-lipid particles (SALP) & $80-140 \mathrm{~nm}$ & $-1,8$ to $-0,8 \mathrm{mV}$ & PEG -Cer & $x$ & $\begin{array}{l}\text { AsODN against ICAM-1, c-myc and c-myb } \\
\text { (in vivo : systemic injection) }\end{array}$ & [213] \\
\hline Lipoplexes (AtuFECT01 + DPhyPE ) & $117 \mathrm{~nm}$ & $+46 \mathrm{mV}$ & DSPE-PEG & $x$ & $\begin{array}{c}\text { siRNA against PTEN } \\
\text { siRNA against CD31 } \\
\text { (in vivo : systemic injection) }\end{array}$ & [211-212] \\
\hline PEGylated gelatin nanoparticles & $290-320 \mathrm{~nm}$ & $-7 \mathrm{mV}$ & $\begin{array}{l}\text { PEG-succinimidyl } \\
\text { glutarate }\end{array}$ & $\mathbf{x}$ & $\begin{array}{c}\mathrm{pDNA} \text { VEGF-R1 } \\
\text { (in vivo : systemic delivery) }\end{array}$ & [201] \\
\hline \multicolumn{7}{|c|}{ Targeted systems } \\
\hline Coated cationic liposomes (CCL) & $70-100 \mathrm{~nm}$ & +7 to $+10 \mathrm{mV}$ & DSPE-PEG & $\begin{array}{l}\text { mAb against-GD2 (Neuroectodermal } \\
\text { tumor) }\end{array}$ & $\begin{array}{l}\text { AsODN against c-myb } \\
\text { (in vivo : systemic injection) }\end{array}$ & [214-215] \\
\hline Sterically stabilized immunolipoplexes & $89 \pm 6.6 \mathrm{~nm}$ & $+9,7$ to $24,1 \mathrm{mV}$ & MAL-PEG-NHS & scFv against -Transferrin receptor & $\begin{array}{c}\text { P53 gene } \\
\text { (in vivo : systemic injection) }\end{array}$ & [238-240] \\
\hline Pegylated PEI & $90 \pm 10 \mathrm{~nm}$ & +5 to $+7 \mathrm{mV}$ & PEG & $\begin{array}{c}\text { RGD peptides } \\
\text { (targeting tumor vasculature) }\end{array}$ & $\begin{array}{c}\text { siRNA (VEGF R2) } \\
\text { (in vivo: systemic injection) }\end{array}$ & [241] \\
\hline Gal-C4-Cholesterol lipoplexes & $141-235 \mathrm{~nm}$ & Not informed & $x$ & $\begin{array}{c}\text { Galactose } \\
\text { (targeting hepatocytes) }\end{array}$ & $\begin{array}{c}\mathrm{pCMVluc} \\
\text { (in vivo : intraportal injection) }\end{array}$ & {$[242,243]$} \\
\hline Lipoplexes-protamine- DNA (LPD) & Not informed & Not informed & DSPE-PEG & $\begin{array}{c}\text { Anisamide (targeting sigma receptor } \\
\text { over-expressed in human lung tumor } \\
\text { cells) }\end{array}$ & $\begin{array}{l}\text { AsODN and siRNA against survivin } \\
\text { (in vitro) }\end{array}$ & [250] \\
\hline PEG/PEI/DNA complexes & $620-640 \mathrm{~nm}$ & $+0,3$ to $4,3 \mathrm{mV}$ & PEG & Tumor-specific CNGRC peptide & $\begin{array}{l}\text { pCMVp53 including SV40 DNTS and NLS binding site } \\
\text { (in vivo : systemic injection) }\end{array}$ & {$[248,294]$} \\
\hline
\end{tabular}


Table 2: Example of promising systems for systemic gene delivery.

mAb: monoclonal antibody, scFv: single-chain antibody fragment, pDNA : plasmidic DNA, asODN: antisens oligodeoxynucleotides, siRNA: small interfering RNA, DSPE-PEG:

1,2-distearoylglycero-3- phosphatidylethanolamine-N- polyethylene glycol, PEG-Cer : PEG-ceramide, PEG-DAG: PEG-Diacylglycerol, PEG-C-DMA: 3-N-

[(methoxypoly(ethyleneglycol) 2000) carbamoyl]-1,2-dimyristyloxy-propylamine, MAL-PEG-NHS: Maleimide-PEG-hydroxysuccinimide, AtuFECT01 : cationic lipid, DPhyPE: 1,2diphytanoyl-sn-glycero-3-phosphoethanolamine, DTNS : DNA nuclear targeting signal, NLS: nuclear localization signal peptide.

SV40 nuclear localization signal peptide

\section{$\mathbf{x}$ : Absence}

* Depending on the method of preparation

** Depending on the $+/$ - charge ratio

${ }^{* \star *}$ The application to cancer therapy is indicated when it exists; otherwise, reporter gene are used (e.g plasmid encoding luciferase, beta galactosidase, green fluorescent protein 
